\title{
Dynamics on Networks of Cluster States for Globally Coupled Phase Oscillators*
}

\author{
Peter Ashwin ${ }^{\dagger}$, Gábor Orosz ${ }^{\dagger}$, John Wordsworth ${ }^{\dagger}$, and Stuart Townley ${ }^{\dagger}$
}

Abstract. Systems of globally coupled phase oscillators can have robust attractors that are heteroclinic networks. We investigate such a heteroclinic network between partially synchronized states where the phases cluster into three groups. For the coupling considered there exist 30 different three-cluster states in the case of five oscillators. We study the structure of the heteroclinic network and demonstrate that it is possible to navigate around the network by applying small impulsive inputs to the oscillator phases. This paper shows that such navigation may be done reliably even in the presence of noise and frequency detuning, as long as the input amplitude dominates the noise strength and the detuning magnitude, and the time between the applied pulses is in a suitable range. Furthermore, we show that, by exploiting the heteroclinic dynamics, frequency detuning can be encoded as a spatiotemporal code. By changing a coupling parameter we can stabilize the three-cluster states and replace the heteroclinic network by a network of excitable three-cluster states. The resulting "excitable network" has the same structure as the heteroclinic network and navigation around the excitable network is also possible by applying large impulsive inputs. We also discuss features that have implications for related models of neural activity.

Key words. globally coupled oscillators, three-cluster state, heteroclinic connection/network, winnerless competition, excitable dynamics, neural computation

AMS subject classifications. 34C15, 37C29, 37G40, 92B20

DOI. $10.1137 / 070683969$

1. Introduction. Coupled phase oscillators are a simple class of dynamical systems that can model phenomena such as synchronization between individual neurons [7, 16]. If neurons are assumed to produce periodic output (e.g., periodic spiking) when uncoupled, then they may be represented by a scalar phase variable. (In case of spiking a phase can be defined such that it crosses zero when the neuron spikes.) Coupled oscillators that are subject to external perturbations can be modeled in many cases by using only the phase variables [8].

Recent work [1] proposes that certain types of neural system are well modeled by what they call winnerless competition. Such dynamics consists of a number of saddle states in the phase space that are connected by their unstable manifolds to form a heteroclinic network. This type of dynamics can robustly produce a number of behaviors in the phase oscillator system that are of interest for modeling neural systems:

(i) it can generate a sequence of states in response to a sequence of inputs,

(ii) it can act as a very sensitive classifier for inputs, and

${ }^{*}$ Received by the editors February 28, 2007; accepted for publication (in revised form) by B. Ermentrout June 5, 2007; published electronically December 7, 2007. This research was supported by the EPSRC grant EP/C510771/1. http://www.siam.org/journals/siads/6-4/68396.html

${ }^{\dagger}$ Mathematics Research Institute, School of Engineering, Computer Science and Mathematics, University of Exeter, EX4 4QF, United Kingdom (p.ashwin@exeter.ac.uk, g.orosz@exeter.ac.uk, j.wordsworth@exeter.ac.uk, s.b. townley@exeter.ac.uk). The last author was supported by the Leverhulme Trust Research Fellowship RF/9/RFG/ $2005 / 0153$. 


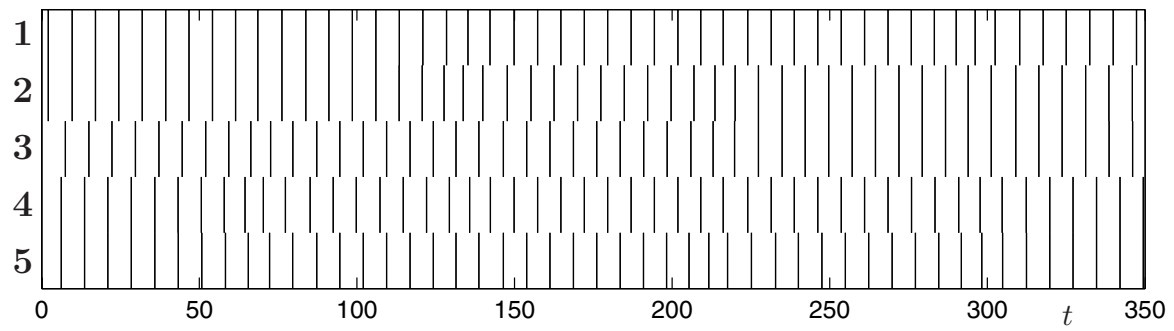

Figure 1. Raster plot showing the spiking of five oscillators in time. The vertical lines indicate when the phases of oscillators $1, \ldots, 5$ pass through zero. The figure is obtained using the coupled phase oscillator model (2.1), (2.2) with parameters (3.1) in the case of low noise (of strength $\eta=10^{-5}$ ), no input $(\epsilon=0)$, and no detuning $(\delta=0)$. Observe that the oscillators form into three-cluster states of two synchronized pairs and one singleton, and that the cluster formations spontaneously change as time progresses. The dynamics in this figure is shown also in Figure 9(a2), where clusters are distinguished by color.

(iii) it can convert inputs into spatiotemporal coding;

see $[6,23]$. The last behavior may have importance for encoding odor information in antennal lobes of insects [11].

Attracting robust heteroclinic networks have been recognized in a variety of systems. For instance, they are found in the Lotka-Volterra dynamics of three or more interacting populations [18], in many symmetric systems [12], and in systems of four or more globally coupled phase oscillators [5] where they cause the phenomena of slow oscillations/switching among cluster states $[13,17,21]$. In case of slow oscillations/switching, the presence of noise causes a near-periodic switching motion such that the asymptotic period of switching becomes unbounded as the noise level reduces to zero.

To motivate our work we present here an example of slow switching. Figure 1 shows a time series of "spikes" for five oscillators which is a widely accepted representation of the dynamics in neural systems [23]. This figure is produced using the phase oscillator model studied in this article with added low noise. Vertical lines show where the phases of oscillators pass through zero. From this "raster plot" the underlying dynamics is not immediately obvious. However, one can recognize that three-cluster states are formed consisting of two synchronized pairs of oscillators and one singleton, and that there are regular switches between these cluster states as time progresses. This figure corresponds to Figure 9(a2), where the clusters are colored yellow and blue (and different time scales are used). Our aim is to show the existence of a heteroclinic network of cluster states which underlies and explains this dynamics. By exploiting the features of this network we can predict possible transitions between cluster states and estimate the period of switching.

Even for quite simple coupling between fairly small numbers of globally coupled phase oscillators one can find attracting robust (structurally stable) heteroclinic networks of high complexity. For example, a system of five oscillators may possess an attractor with 20 different two-cluster states linked together as a heteroclinic network [2]. This network can be used to perform simple computations by applying small inputs to the individual oscillators [3], but these computations are very sensitive to noise. For this reason, we introduce a modified model in section 2 which presents a new type of heteroclinic network between three-cluster states and where the performed computations are considerably less sensitive to noise. The goal of 
this paper is to describe the structure and dynamics of this network and also to investigate its computational properties.

In section 3 we examine the structure of the proposed heteroclinic network in detail. For five oscillators we find an open set of parameters such that the system has a robust heteroclinic attractor comprising 30 symmetrically related three-cluster states of saddle type connected by their unstable manifolds. We also investigate the behavior of the network under perturbations. Namely, we consider the effect of noise, the effect of detuning the natural frequencies of the oscillators, and the effect of (periodic) impulsive inputs.

In section 4 we demonstrate that the heteroclinic network can robustly perform finitestate computation in the presence of background noise and frequency detuning. By applying periodic impulsive inputs to the phase oscillators it is possible to induce switches between cluster states and so predictably navigate around the network. We characterize the bit error rate of these switches as a function of the input period, the input amplitude, and the noise strength. It is shown that as long as the input amplitude is large enough (compared to the noise strength) there exists a range of periods for which the computations are reliable. The left boundary of this range is proportional to the logarithm of the input amplitude, while its right boundary is proportional to the logarithm of the noise strength. (Frequency detuning also brings errors into the finite-state computation similarly to noise.) At the end of section 4 we also discuss that frequency detuning can be encoded as spatiotemporal codes.

Section 5 shows that on varying a single parameter in the coupling function one can achieve that the above attracting heteroclinic network becomes an attracting network of linearly stable three-cluster states. These states are excitable: there is a minimum threshold for the input amplitude to switch from one state to the other. This "excitable network" can perform finitestate computation similarly to the heteroclinic network, except that the input amplitudes must be sufficiently large.

We conclude our results in section 6 and discuss future research in section 7 .

2. The phase oscillator model. In this section we introduce the globally coupled phase oscillator model we study in this paper. The first model of this kind was developed by Kuramoto [19], but models with several different coupling functions have been investigated in the last three decades; see, e.g., $[2,7,13]$. We consider $N$ oscillators complying with the equations

$$
\dot{\theta_{n}}=\omega_{n}+\frac{1}{N} \sum_{m=1}^{N} g\left(\theta_{n}-\theta_{m}\right)+\epsilon I_{n}(t)+\eta w_{n}, \quad n=1, \ldots, N
$$

where $\theta_{n}(t)=\theta_{n}(t+2 \pi)$ is the phase of the $n$th oscillator, $\omega_{n}$ is the natural frequency of the $n$th oscillator, $I_{n}(t)$ is an impulsive input with unit magnitude, and $w_{n}(t)$ is uncorrelated white noise such that the associated random walk has unit growth of variance per unit time. The quantity $\epsilon$ represents the input amplitude, while $\eta$ is the noise strength. The globally coupled system (2.1), (2.2) is represented by the graphs in Figure 2 for $N=3,5,7$ oscillators (black dots) with $N(N-1) / 2=3,10,21$ bidirectional connections (two-headed arrows). We remark that we are interested only in the case when $N$ is finite. In particular, $N=5$ is studied in detail, and implications for larger $N$ are discussed in section 7 . In some cases we will use the 

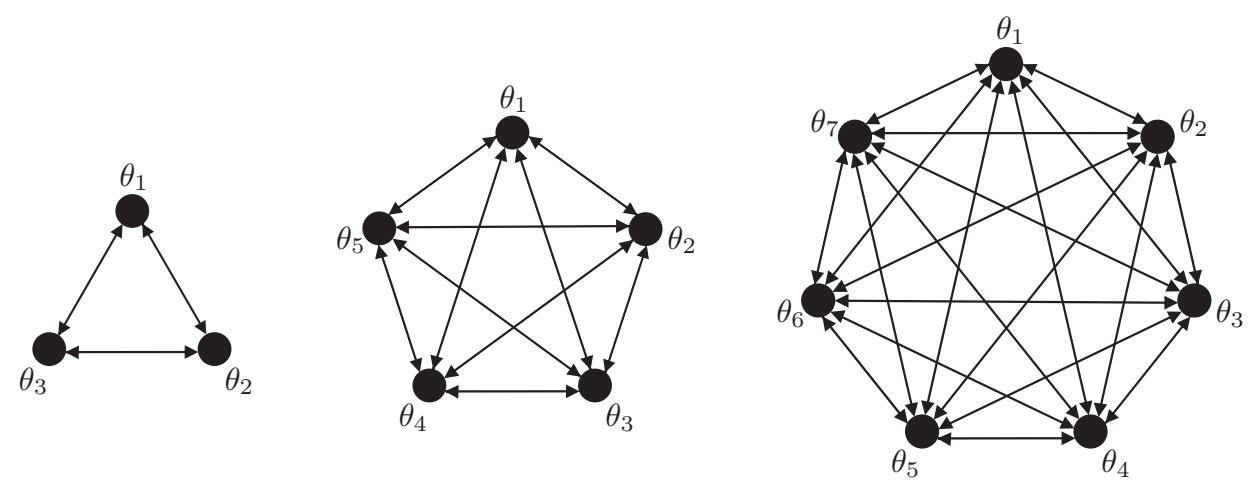

Figure 2. Global coupling between $N=3,5,7$ oscillators with $N(N-1) / 2=3,10,21$ bidirectional connections, respectively. Black dots represent the oscillators, and two-headed arrows represent the mutual coupling between them.

vector notation $\theta(t)=\operatorname{col}\left[\theta_{1}(t), \ldots, \theta_{N}(t)\right]$. Furthermore, we consider a coupling function

$$
g(\varphi)=-\sin (\varphi+\alpha)+r \sin (2 \varphi+\beta),
$$

where $\alpha, \beta$, and $r$ are constants. This function is $2 \pi$-periodic containing Fourier modes with frequencies 1 and 2 . Note that in order to find nontrivial clustering behavior in the system (2.1), (2.2) it is necessary to include Fourier modes with frequencies higher than 1 [13]. Furthermore, the phase reductions of well-known neural models naturally lead to periodic functions where several harmonics are included [14]. Function (2.2) is the simplest generalization of the functions used in $[2,13,17]$.

One may define the average natural frequency by

$$
\omega=\frac{1}{N} \sum_{n=1}^{N} \omega_{n},
$$

and a uniform detuning of natural frequencies is when

$$
\omega_{n+1}-\omega_{n}=\delta, \quad n=1, \ldots, N-1 .
$$

Here $\delta \ll 1$ represents the detuning magnitude. Observe that for uniform detuning given $\omega$ and $\delta$ we have

$$
\omega_{n}=\omega+\left(n-\frac{N+1}{2}\right) \delta, \quad n=1, \ldots, N .
$$

Permuting the oscillators in (2.4), and consequently in (2.5), gives detunings which we also call uniform. In the case of nonuniform detunings the frequencies $\omega_{n}$ are not uniformly spaced and the detuning magnitude may be defined by

$$
\delta=\max _{n}\left\{\min _{m \neq n}\left\{\left|\omega_{n}-\omega_{m}\right|\right\}\right\} .
$$

In this article we investigate only the effects of uniform detunings. Note that $\delta=0$ if and only if $\omega_{n}=\omega$ for $n=1, \ldots, N$. 
One may consider the detuning as a steady external input applied to the "ideal" system of identical oscillators $\left(\omega_{n}=\omega\right.$ for all $\left.n\right)$ in addition to impulsive inputs and noise in (2.1). The effects of the external perturbations in the form of impulsive inputs (when $\epsilon \neq 0$ ), noise (when $\eta \neq 0$ ), and detuning (when $\delta \neq 0$ ) will be discussed in section 3.3.

In the "unperturbed" case

$$
\epsilon=\eta=\delta=0
$$

the systems of coupled oscillators $(2.1),(2.2)$ can be thought of as ODEs on the $N$-torus $\mathbb{T}^{N}$ with symmetry $S_{N}$ of all permutations of the oscillators. The codimension one invariant subspaces

$$
\theta_{n}(t) \equiv \theta_{m}(t) \quad \text { for } \quad n \neq m
$$

form barriers to the flow. These divide the torus up into $(N-1)$ ! invariant regions that are symmetric images of the canonical invariant region

$$
\left\{\theta_{1} \leq \theta_{2} \leq \cdots \leq \theta_{N} \leq \theta_{1}+2 \pi\right\}
$$

see [5] for more details. Subspaces (2.8) are essential for the formation of the robust heteroclinic network, as will be explained in section 3.1.

We refer to an $\ell$-cluster state of type $\left(N_{1}, \ldots, N_{\ell}\right)$ as a state where each oscillator has one of only $\ell$ different phases $\phi_{1}, \ldots, \phi_{\ell}$ : for each $r$ there are $N_{r}$ oscillators that have the same phase $\phi_{r}$ such that $\sum_{r=1}^{\ell} N_{r}=N$. In this way we can think of full synchrony as a one-cluster state of type $(N)$ and full asynchrony as an $N$-cluster state of type $(1, \ldots, 1)$ with $N$ entries. Note that choosing a clustered distribution of frequencies $\omega_{n}$ can give rise to cluster states of similar type. However, this is not necessary as cluster states may appear spontaneously as stable behavior, even for identical oscillators $\left(\omega_{n}=\omega\right.$ for all $\left.n\right)$, as will be discussed in detail in section 5 .

We wish to emphasize the importance of the parameter $\beta$ in the coupling function (2.2). In $[2,13,17] \beta=0$ was considered; i.e., only the odd part of the second harmonic was included. Due to this "degeneracy" of the coupling function it appears that any attracting heteroclinic network contains only two-cluster states. In this paper we unfold the above "degeneracy" by considering $\beta \neq 0$ and find heteroclinic networks between three-cluster states.

Because the right-hand side of (2.1) depends only on phase differences, there is an extra $S^{1}$ continuous rotational symmetry given by performing an identical phase shift to all oscillators. If there is a solution $\tilde{\theta}(t)=\operatorname{col}\left[\tilde{\theta}_{1}(t), \ldots, \tilde{\theta}_{N}(t)\right]$ of $(2.1)$ then $\tilde{\theta}(t)+\varphi \operatorname{col}[1, \ldots, 1]=$ $\operatorname{col}\left[\tilde{\theta}_{1}(t)+\varphi, \ldots, \tilde{\theta}_{N}(t)+\varphi\right]$ is also a solution for any $\varphi \in \mathbb{R}$. Consequently, it is sufficient to examine phase differences to determine the long-term behavior of the system. We will introduce the phase differences $\gamma_{n}=\theta_{n}-\theta_{N}$ for $n=1, \ldots, N$. Note that similar continuous symmetries appear in many other applications, e.g., in car-following models of highway traffic [22].

3. Attracting network of three-cluster states. In the unperturbed case $\epsilon=\eta=\delta=0$ system (2.1), (2.2) displays a wide range of robust dynamics. This includes synchrony ( $r, \alpha$ small regardless of $\beta$ ), antisynchrony ( $r$ small $\alpha$ near $\pi$ regardless of $\beta$ ), and cluster states (typically in the region where $\alpha$ is close to $\pi / 2$ ). In the last case one can find robust heteroclinic 

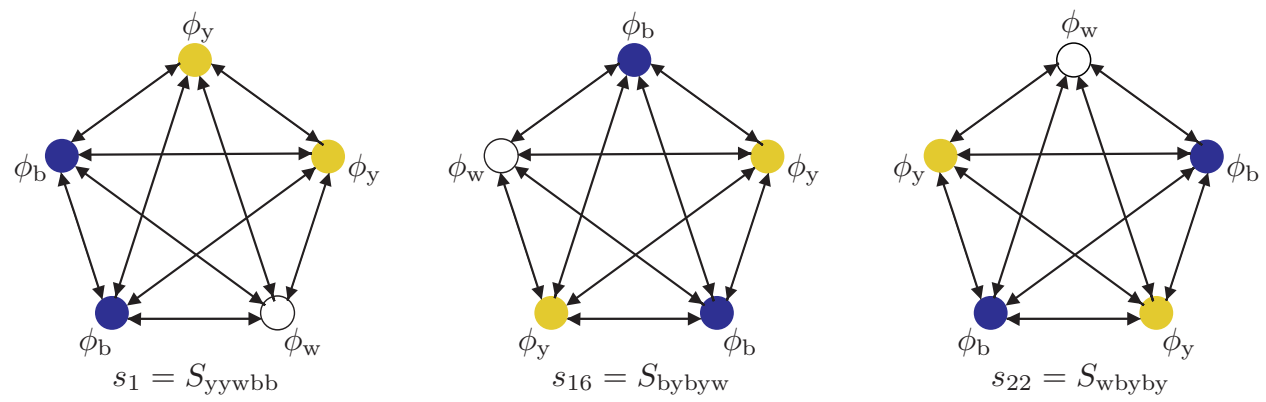

Figure 3. Three of the 30 three-cluster states of Table 1 are represented by color. The pairs marked " $\mathrm{y}$ " are shown as yellow, the pairs marked "b" are shown as blue, and the single oscillators are displayed as white.

connections. For $\beta=0$ these connections are between two-cluster states [2, 17], but the system with $\beta \neq 0$ can have connections between three-cluster states.

Considering $N=5$ the system (2.1), (2.2) has an attracting heteroclinic network for an open set of parameters near

$$
r=0.2, \quad \alpha=1.8, \quad \beta=-2.0, \quad \omega=1.0,
$$

as explained below. Considering the invariant subspace

$$
\left[\begin{array}{l}
\theta_{1}(t) \\
\theta_{2}(t) \\
\theta_{3}(t) \\
\theta_{4}(t) \\
\theta_{5}(t)
\end{array}\right]=\left[\begin{array}{l}
\theta_{1}(t) \\
\theta_{1}(t) \\
\theta_{3}(t) \\
\theta_{4}(t) \\
\theta_{4}(t)
\end{array}\right]
$$

where the first two oscillators form a cluster $\left(\theta_{1}(t) \equiv \theta_{2}(t)\right)$ and the last two oscillators form another cluster $\left(\theta_{4}(t) \equiv \theta_{5}(t)\right)$, one can find a three-cluster state of type $(2,1,2)$

$$
\left[\begin{array}{l}
\theta_{1}(t) \\
\theta_{2}(t) \\
\theta_{3}(t) \\
\theta_{4}(t) \\
\theta_{5}(t)
\end{array}\right]=\left[\begin{array}{l}
\Omega t+\phi_{\mathrm{y}} \\
\Omega t+\phi_{\mathrm{y}} \\
\Omega t+\phi_{\mathrm{w}} \\
\Omega t+\phi_{\mathrm{b}} \\
\Omega t+\phi_{\mathrm{b}}
\end{array}\right]:=S_{\mathrm{yywbb}}
$$

for some constants $\phi_{\mathrm{y}}<\phi_{\mathrm{w}}<\phi_{\mathrm{b}}<\phi_{\mathrm{y}}+2 \pi$. Note that the subscripts of $S_{\mathrm{yywbb}}$ correspond to the subscripts of the coordinates where "y," "w," and "b" stand for yellow, white, and blue, respectively; see the coloring applied in the leftmost panel of Figure 3. In this article we refer to this coloring by using terms such as yellow oscillator/cluster, white oscillator, and blue oscillator/cluster.

In addition there are a number of states given by permutation of the subscripts of $S_{\text {yywbb }}$, that is, by permutation of the coordinates in (3.3). This symmetry gives a total of $5 ! /(2 ! 1 ! 2 !)=$ 30 possible states listed in Table 1. Some of these states are also represented by the graphs in Figure 3 with corresponding coloring. 
Table 1

List of three-cluster states. Each column may be generated from another by cyclic permutation of the subscripts of $S_{i_{1}, \ldots, i_{5}}$.

\begin{tabular}{|c|r|r|r|r|}
\hline$s_{1}=S_{\text {yywbb }}$ & $s_{7}=S_{\text {byywb }}$ & $s_{13}=S_{\text {bbyyw }}$ & $s_{19}=S_{\text {wbby }}$ & $s_{25}=S_{\text {ywbby }}$ \\
$s_{2}=S_{\text {bbwyy }}$ & $s_{8}=S_{\text {ybbwy }}$ & $s_{14}=S_{\text {yybbw }}$ & $s_{20}=S_{\text {wyybb }}$ & $s_{26}=S_{\text {bwyyb }}$ \\
$s_{3}=S_{\text {ybwyb }}$ & $s_{9}=S_{\text {bybwy }}$ & $s_{15}=S_{\text {ybybw }}$ & $s_{21}=S_{\text {wybyb }}$ & $s_{27}=S_{\text {bwyby }}$ \\
$s_{4}=S_{\text {bywby }}$ & $s_{10}=S_{\text {ybywb }}$ & $s_{16}=S_{\text {bybyw }}$ & $s_{22}=S_{\text {wbyb }}$ & $s_{28}=S_{\text {ywbyb }}$ \\
$s_{5}=S_{\text {ybwby }}$ & $s_{11}=S_{\text {yybwb }}$ & $s_{17}=S_{\text {byybw }}$ & $s_{23}=S_{\text {wbyy }}$ & $s_{29}=S_{\text {bwbyy }}$ \\
$s_{6}=S_{\text {bywyb }}$ & $s_{12}=S_{\text {bbywy }}$ & $s_{18}=S_{\text {ybbyw }}$ & $s_{24}=S_{\text {wybby }}$ & $s_{30}=S_{\text {ywybb }}$ \\
\hline
\end{tabular}

Notice that the three-cluster state (3.3) (and each symmetric copy in Table 1) has full frequency synchrony with frequency $\Omega$ and clustering into three-cluster states with phases $\phi_{\mathrm{y}}$, $\phi_{\mathrm{w}}$, and $\phi_{\mathrm{b}}$. One of these phases can be chosen arbitrarily corresponding to the $S^{1}$ continuous rotational symmetry of the system. This means that the three-cluster state (3.3) (and each symmetric copy in Table 1) is determined by the phase differences

$$
\begin{aligned}
& \chi:=\phi_{\mathrm{y}}-\phi_{\mathrm{w}}, \quad \text { where }-\pi<\chi<0, \\
& \psi:=\phi_{\mathrm{b}}-\phi_{\mathrm{w}}, \quad \text { where } 0<\psi<\pi,
\end{aligned}
$$

up to application of the continuous rotational symmetry. Substituting the solution (3.3) into (2.1), (2.2) when $\epsilon=\eta=\delta=0$ and using (3.4), one obtains

$$
\begin{aligned}
& \Omega=\omega+\frac{1}{5}(g(0)+2 g(-\chi)+2 g(-\psi)), \\
& \Omega=\omega+\frac{1}{5}(2 g(0)+g(\chi)+2 g(\chi-\psi)), \\
& \Omega=\omega+\frac{1}{5}(2 g(0)+g(\psi)+2 g(\psi-\chi)),
\end{aligned}
$$

which determine $\chi, \psi$, and $\Omega$. Note that when solving (3.5) the frequency $\omega$ does not appear in the phase differences $\chi$ and $\psi$. For parameters (3.1) there is a unique solution:

$$
\chi=-1.8212, \quad \psi=1.1041, \quad \Omega=0.8468 .
$$

Linearizing system (2.1), (2.2) about the three-cluster state $s_{1}=S_{\text {yywbb }}$ in (3.3) (or about any symmetric copy in Table 1) and using (3.4), one can investigate the linear stability of three-cluster states, that is, determine the eigenvalues

$$
\begin{aligned}
& \lambda_{1}=0, \\
& \lambda_{2}=\frac{1}{5}\left(2 g^{\prime}(0)+g^{\prime}(\chi)+2 g^{\prime}(\chi-\psi)\right), \\
& \lambda_{3}=\frac{1}{5}\left(2 g^{\prime}(0)+g^{\prime}(\psi)+2 g^{\prime}(\psi-\chi)\right), \\
& \lambda_{4}=\lambda_{5}^{*}=\mu+\mathrm{i} \sqrt{\nu}
\end{aligned}
$$

of the linearized system. The rather complicated expressions of $\mu, \nu \in \mathbb{R}^{+}$in terms of $g^{\prime}(\chi)$, $g^{\prime}(-\chi), g^{\prime}(\psi), g^{\prime}(-\psi), g^{\prime}(\chi-\psi)$, and $g^{\prime}(\psi-\chi)$ are given by (A.1), (A.2) in Appendix A. For parameters (3.1) (that is, for the phase differences (3.6)) we have the eigenvalues

$$
\lambda_{2}=-0.2834, \quad \lambda_{3}=0.1703, \quad \lambda_{4,5}=-0.1012 \pm \mathrm{i} 0.2848,
$$

Copyright $@$ by SIAM. Unauthorized reproduction of this article is prohibited. 


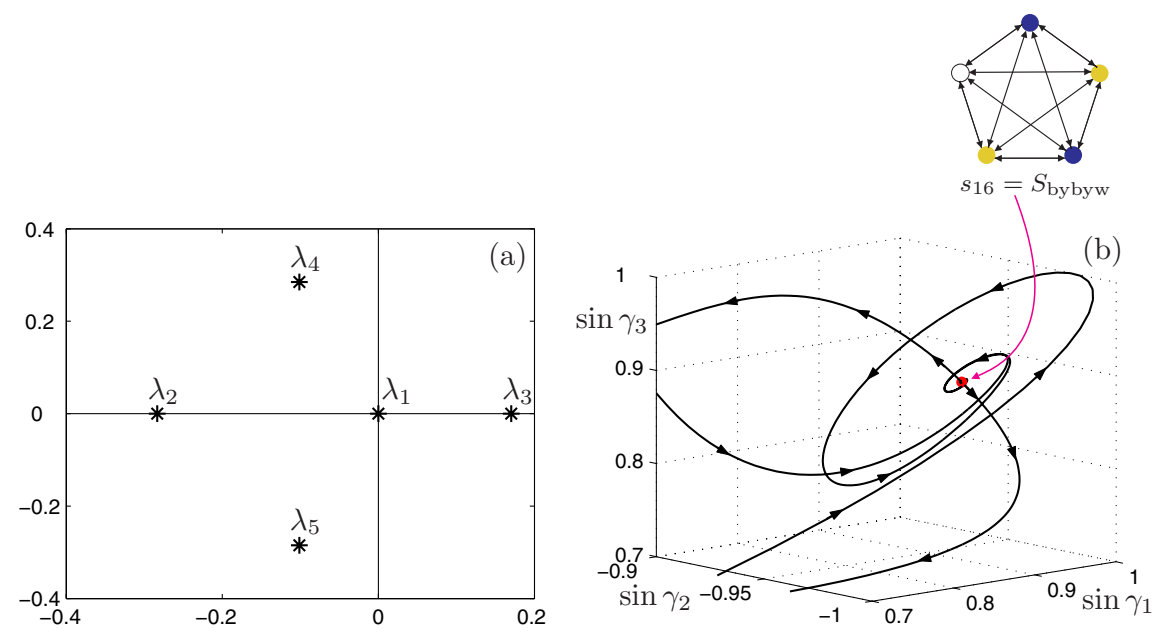

Figure 4. Dynamics around a saddle three-cluster state for parameters (3.1) in the unperturbed case $\epsilon=\eta=\delta=0$. In panel (a) the eigenvalues (3.7), (3.8) are displayed in the complex plane. Panel (b) shows the local dynamics in phase space in the vicinity of the three-cluster state $s_{16}=S_{\text {bybyw }}$ (red dot): The unstable eigendirection $v_{3}$ and the stable eigendirections $\operatorname{Re}\left(v_{4}\right)=\operatorname{Re}\left(v_{5}\right), \operatorname{Im}\left(v_{4}\right)=-\operatorname{Im}\left(v_{5}\right)$ given in (3.9) can be visualized by the outgoing and spiralling-in trajectories, respectively.

shown in Figure 4(a). For the three-cluster state $s_{1}=S_{\text {yywbb }}$ the corresponding eigenvectors are

$$
v_{1}=\left[\begin{array}{l}
1 \\
1 \\
1 \\
1 \\
1
\end{array}\right], \quad v_{2}=\left[\begin{array}{c}
1 \\
-1 \\
0 \\
0 \\
0
\end{array}\right], \quad v_{3}=\left[\begin{array}{c}
0 \\
0 \\
0 \\
1 \\
-1
\end{array}\right], \quad v_{4}=v_{5}^{*}=\left[\begin{array}{c}
r_{\mathrm{y}}+\mathrm{i} p_{\mathrm{y}} \\
r_{\mathrm{y}}+\mathrm{i} p_{\mathrm{y}} \\
1 \\
r_{\mathrm{b}}+\mathrm{i} p_{\mathrm{b}} \\
r_{\mathrm{b}}+\mathrm{i} p_{\mathrm{b}}
\end{array}\right] \text {, }
$$

where the expressions of $r_{\mathrm{y}}, p_{\mathrm{y}}, r_{\mathrm{b}}, p_{\mathrm{b}} \in \mathbb{R}$ contain $g^{\prime}(\chi), g^{\prime}(-\chi), g^{\prime}(\psi), g^{\prime}(-\psi), g^{\prime}(\chi-\psi)$, and $g^{\prime}(\psi-\chi)$ as given by (A.3)-(A.6) in Appendix A. For the other 29 states in Table 1, the eigenvectors can be obtained by permuting the components in (3.9) (in the same manner as the subscripts of $s_{1}=S_{\text {yywbb }}$ to obtain these symmetric copies).

In order to eliminate the $S^{1}$ continuous rotational symmetry we use the phase differences

$$
\left[\begin{array}{c}
\gamma_{1} \\
\gamma_{2} \\
\gamma_{3} \\
\gamma_{4} \\
\gamma_{5}
\end{array}\right]=\left[\begin{array}{c}
\theta_{1}-\theta_{5} \\
\theta_{2}-\theta_{5} \\
\theta_{3}-\theta_{5} \\
\theta_{4}-\theta_{5} \\
0
\end{array}\right]
$$

to plot the dynamics of the system. In the phase space of these coordinates the three-cluster state $s_{1}=S_{\text {yywbb }}$ in (3.3) and its symmetrical copies in Table 1 are equilibria. Furthermore, in many of the subsequent figures we project the phase space $\mathbb{T}^{4}$ of the phase differences $\gamma_{n}$ into $[-1,1]^{4}$ by taking $\sin \gamma_{n}$. 
The eigenvector $v_{1}$ with zero eigenvalue corresponds to translation along the $S^{1}$ group orbit of the system. The eigenvectors $v_{2}$ and $v_{3}$ correspond to splitting the yellow and the blue clusters, respectively. (These clusters are marked by subscripts "y" and "b" in (3.3) and Table 1, and are colored yellow and blue in Figures 3 and 4.) The eigenvectors $v_{4}$ and $v_{5}$ correspond to motion in the subspace (3.2). In Figure 4(b) the eigendirection $v_{3}$ and the eigendirections $\operatorname{Re}\left(v_{4}\right)=\operatorname{Re}\left(v_{5}\right), \operatorname{Im}\left(v_{4}\right)=-\operatorname{Im}\left(v_{5}\right)$ can be visualized by the outgoing and spiralling-in trajectories, respectively, for the three-cluster state $s_{16}=S_{\text {bybyw }}$ (red dot).

For parameters (3.1) we have $\lambda_{1}=0, \lambda_{2}<0, \lambda_{3}>0$, and $\operatorname{Re}\left(\lambda_{4}\right)=\operatorname{Re}\left(\lambda_{5}\right)<0$ as given by (3.8) and plotted in Figure 4(a). Consequently, the eigendirection $v_{2}$ is attracting; that is, perturbations that involve splitting the yellow cluster will decay in time. Hence we say that the yellow cluster is stable. On the other hand, the eigendirection $v_{3}$ is repelling as the outgoing trajectories demonstrate in Figure 4(b). That is, perturbations that involve splitting the blue cluster grow in time. Hence we say that the blue cluster is unstable. The eigendirections $\operatorname{Re}\left(v_{4}\right)=\operatorname{Re}\left(v_{5}\right)$ and $\operatorname{Im}\left(v_{4}\right)=-\operatorname{Im}\left(v_{5}\right)$ are attracting, as demonstrated by the inward spiralling trajectories in Figure 4(b). That is, the three-cluster state is attracting in the subspace (3.2). In summary, the only perturbations giving rise to motion away from the saddle three-cluster state are those in the direction $v_{3}$ that involve splitting the blue cluster.

3.1. Heteroclinic connections between three-cluster states. One can verify numerically that the branches of the one-dimensional unstable manifold of the three-cluster state $s_{1}=$ $S_{\text {yywbb }}$ are contained wholly within the stable manifolds of the three-cluster states $s_{12}=$ $S_{\text {bbywy }}$ and $s_{13}=S_{\text {bbyyw }}$. The former branch corresponds to the direction where the 4 th oscillator is advanced relative to the 5 th one, i.e., "starts in direction $v_{3}$," while the latter branch corresponds to the opposite direction, i.e., "starts in direction $-v_{3}$ "; see (3.9). These connections occur within the invariant subspace

$$
\left[\begin{array}{l}
\theta_{1}(t) \\
\theta_{2}(t) \\
\theta_{3}(t) \\
\theta_{4}(t) \\
\theta_{5}(t)
\end{array}\right]=\left[\begin{array}{l}
\theta_{1}(t) \\
\theta_{1}(t) \\
\theta_{3}(t) \\
\theta_{4}(t) \\
\theta_{5}(t)
\end{array}\right]
$$

and the connections are source-to-sink and hence robust (persistent) to sufficiently small symmetry-preserving perturbations. Note that on the connecting orbit the first two oscillators remain synchronized $\left(\theta_{1}(t) \equiv \theta_{2}(t)\right.$ ), but this cluster becomes unstable (changes from yellow to blue).

Similarly, one branch of the one-dimensional unstable manifold of each of the three-cluster states $s_{19}=S_{\text {wbbyy }}$ and $s_{29}=S_{\text {bwbyy }}$ connects to $s_{1}=S_{\text {yywbb }}$. These branches "arrive from directions $\mp v_{2}$," and they are embedded in the invariant subspace

$$
\left[\begin{array}{l}
\theta_{1}(t) \\
\theta_{2}(t) \\
\theta_{3}(t) \\
\theta_{4}(t) \\
\theta_{5}(t)
\end{array}\right]=\left[\begin{array}{l}
\theta_{1}(t) \\
\theta_{2}(t) \\
\theta_{3}(t) \\
\theta_{4}(t) \\
\theta_{4}(t)
\end{array}\right]
$$




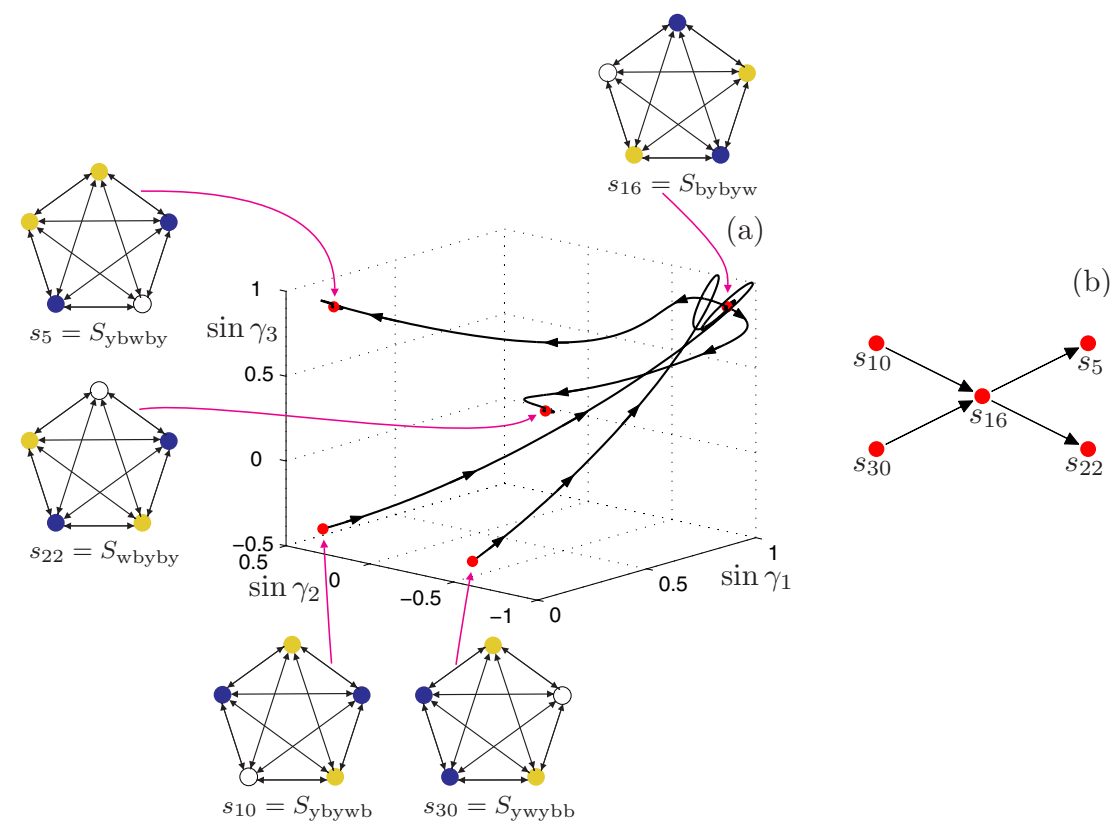

Figure 5. In panel (a) the heteroclinic connections originated in and terminated at the three-cluster state $s_{16}=S_{\text {bybyw }}$ are shown in phase space for parameters (3.1) in the unperturbed case $\epsilon=\eta=\delta=0$. The three-cluster states are represented by red dots. In panel (b) a simple graph representation is displayed such that the nodes and directed edges correspond to three-cluster states and heteroclinic connections, respectively.

where the last two oscillators are synchronized $\left(\theta_{4}(t) \equiv \theta_{5}(t)\right)$.

Indeed, the above argument can be interpreted for any three-cluster state in Table 1. For example, considering the three-cluster state $s_{16}=S_{\text {bybyw }}$, one may verify that the branches of its unstable manifold connect to the three-cluster states $s_{5}=S_{\text {ybwby }}$ and $s_{22}=S_{\text {wbyby }}$, while the unstable manifolds of the three-cluster states $s_{10}=S_{\text {ybywb }}$ and $s_{30}=S_{\text {ywybb }}$ connect to it, as shown in Figure 5(a). The connected three-cluster states can be represented as nodes, while the connections can be represented as directed edges between them, as shown by the graph in Figure 5(b).

3.2. Graph structure of the heteroclinic network. Let us now examine all connections between three-cluster states in Table 1 . We find that there is a heteroclinic network consisting of the 30 three-cluster states $s_{i}=S_{i_{1} \ldots i_{5}}$ and their 60 connecting heteroclinic orbits; see Figure 6(a), where all states and connections are shown in phase space. The network can be represented as the directed graph shown in Figure 6(b). The nodes $s_{i}$ of the graph correspond to the three-cluster states $s_{i}=S_{i_{1} \ldots i_{5}}$, and there is a directed edge from node $s_{i}$ to $s_{j}$ if and only if there is connecting orbit from the three-cluster state $s_{i}=S_{i_{1} \ldots i_{5}}$ to $s_{j}=S_{j_{1} \ldots j_{5}}$. Notice the five-fold cyclic symmetry of the graph that reflects a five-fold cyclic permutation symmetry of the oscillators; see the middle panel in Figure 2.

Starting at a certain three-cluster state of the heteroclinic network in Figure 6(a) (or equivalently at the corresponding node of the graph in Figure 6(b)), we can choose which connection (or corresponding edge) we wish to follow. More precisely, an arbitrarily small perturbation with a component in the direction $v_{3}$ or $-v_{3}$ initiates a transition along one or 

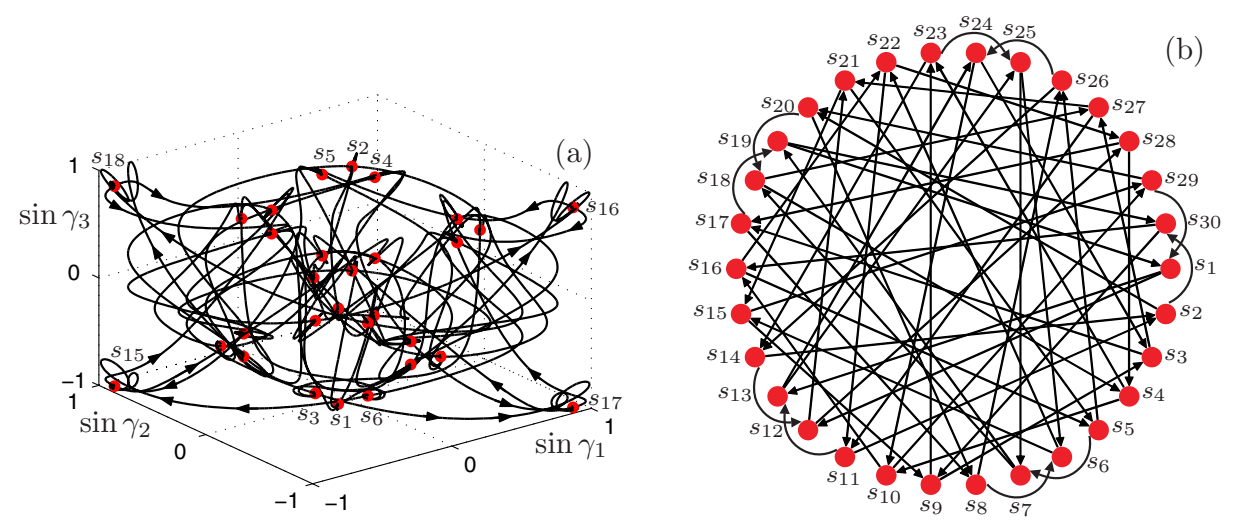

Figure 6. Panel (a) shows the entire heteroclinic network in phase space for parameters (3.1) in the unperturbed case $\epsilon=\eta=\delta=0$. Some of the three-cluster states (red dots) are labeled according to Table 1 . In panel (b) the graph representation is depicted where each node represents a three-cluster state and each directed edge represents a heteroclinic connection from one state to the other.

the other heteroclinic connection. Indeed, these perturbations correspond to advancing one oscillator of the unstable blue cluster (marked by "b" in the subscript of $s_{i}=S_{i_{1} \ldots i_{5}}$ in (3.3) and Table 1 and shown as blue in Figures 3-5). This means that a small advance of phase of the $p$ th oscillator gives rise to a transition from $s_{i}$ to $s_{j}$ if $i_{p}=\mathrm{b}$ (we perturb the unstable blue cluster) and there exists a heteroclinic connection between the three-cluster states $s_{i}=S_{i_{1}, \ldots, i_{5}}$ and $s_{j}=S_{j_{1}, \ldots, j_{5}}$. To formalize this we define operator $O_{p}$ such that

$$
O_{p}\left(s_{i}\right)=s_{j} \quad \Leftrightarrow \quad O_{p}\left(S_{i_{1} \ldots i_{5}}\right)=S_{j_{1} \ldots j_{5}}
$$

if and only if there is a transition from $s_{i}$ to $s_{j}$ obtained by a small advance of the phase of the $p$ th oscillator. The action of $O_{p}$ is characterized by the subscript changes:

$$
\text { if } i_{p}=\mathrm{b}, \quad \text { then }\left\{\begin{array}{l}
i_{n}=\mathrm{y} \rightarrow j_{n}=\mathrm{b}, \\
i_{n}=\mathrm{w} \rightarrow j_{n}=\mathrm{y}, \\
i_{n}=\mathrm{b} \rightarrow j_{n}=\mathrm{y} \text { for } n \neq p, \\
i_{n}=\mathrm{b} \rightarrow j_{n}=\mathrm{w} \text { for } n=p,
\end{array}\right.
$$

According to (3.14) there are two "qualitatively" different choices of $p \in\{1, \ldots, 5\}$. Choosing an oscillator from the unstable blue cluster $\left(i_{p}=\mathrm{b}\right)$ gives $O_{p}\left(s_{i}\right)=s_{j}$ with $j \neq i$, while choosing an oscillator from the stable yellow cluster $\left(i_{p}=\mathrm{y}\right)$ or choosing the single white oscillator $\left(i_{p}=\mathrm{w}\right)$ gives $O_{p}\left(s_{i}\right)=s_{i}$. Formula (3.14) evaluated for all $s_{i}$ is presented as Table 2 in Appendix B. According to this, one can follow arbitrary paths on the directed graph in Figure 6(b) by making a sequence of arbitrarily small perturbations to the appropriate oscillators. We will exploit this feature of the network in sections 3.3 and 4 .

We can define an adjacency matrix $A$ with elements $A_{i j} \in\{0,1\}$, where $A_{i j}=1$ if and only if there is a directed edge from node $s_{i}$ to $s_{j}$ in the graph Figure 6(b). The number 


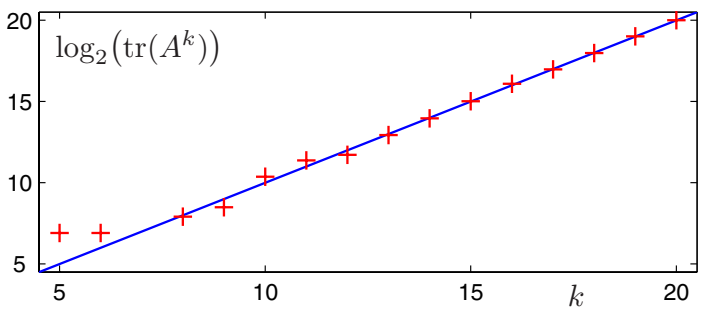

Figure 7. The logarithm of the number of distinct cyclic paths $\log _{2}\left(\operatorname{tr}\left(A^{k}\right)\right)$ as a function of the path length $k$ (red crosses) for the graph shown in Figure 6(b). Note that the shortest cycle length is 5 and there are no cycles of length 7 . The blue line corresponds to the growth $2^{k}$.

of cyclic paths of any given length can be calculated from the adjacency matrix as follows. The elements of the matrix $A^{k}$ give the number of distinct paths of length $k$ from node $s_{i}$ to node $s_{j}$; see [15]. Thus, the diagonal elements of $A^{k}$ give the number of distinct cyclic paths of length $k$ from a node back to itself, and the trace $\operatorname{tr}\left(A^{k}\right)$ represents the total number of cyclic paths of length $k$ in the network. In Figure 7 the logarithm of $\operatorname{tr}\left(A^{k}\right)$ is plotted as a function of $k$ (red crosses). One can observe that there are no cycles of length $1,2,3,4,7$ and that $\operatorname{tr}\left(A^{k}\right) \approx 2^{k}$ for large $k$ (the blue line represents the exact relation $\operatorname{tr}\left(A^{k}\right)=2^{k}$ ). We remark that cycles with path length $k=6$ become relevant when the natural frequencies of the oscillators are detuned in (2.1) according to (2.5) with $\delta \neq 0$, as will be discussed in sections 3.3 and 4.2 .

3.3. Dynamics of the attracting heteroclinic network. For parameters (3.1) in the unperturbed case $\epsilon=\eta=\delta=0$ the heteroclinic network described above is asymptotically stable/attracting (even though each three-cluster state is saddle). (Recall that the contracting eigenvalue is stronger than the expanding one, i.e., $\left|\lambda_{2}\right|>\lambda_{3}$ as given by (3.8) and plotted in Figure 4(a); so by [18] the network is asymptotically stable.) This attractivity is essential if one wishes to perform reliable computations by using the heteroclinic network: small perturbations do not drive the system away from the network as time progresses, allowing navigation along a chosen path on the network.

In Figure 8 the attractivity is demonstrated for an arbitrary initial condition. Figure 8(a) shows the dynamics in phase space. The trajectory approaches the network such that it approaches a sequence of three-cluster states (labeled red dots) and "travels close" to the heteroclinic connections from one state to the other while spending longer and longer periods of time close to the three-cluster states. This well-known slowing down of cycling [18] is seen in time profiles in Figure 8(b), where the plateaux correspond to "being close to" the threecluster states and the quick transitions between them correspond to "traveling close to" the heteroclinic connections. In this panel a color code is also applied: yellow oscillators form the stable cluster, blue oscillators form the unstable cluster, and labels on the top identify the three-cluster states according to Table 1. Regions of quick transitions are colored light green. The length of time intervals spent close to the three-cluster states, i.e., the length of the plateaux, increases as

$$
\lim _{k \rightarrow \infty} \frac{T_{k}}{T_{k-1}}=\frac{\left|\lambda_{2}\right|}{\lambda_{3}}
$$

Copyright $@$ by SIAM. Unauthorized reproduction of this article is prohibited. 


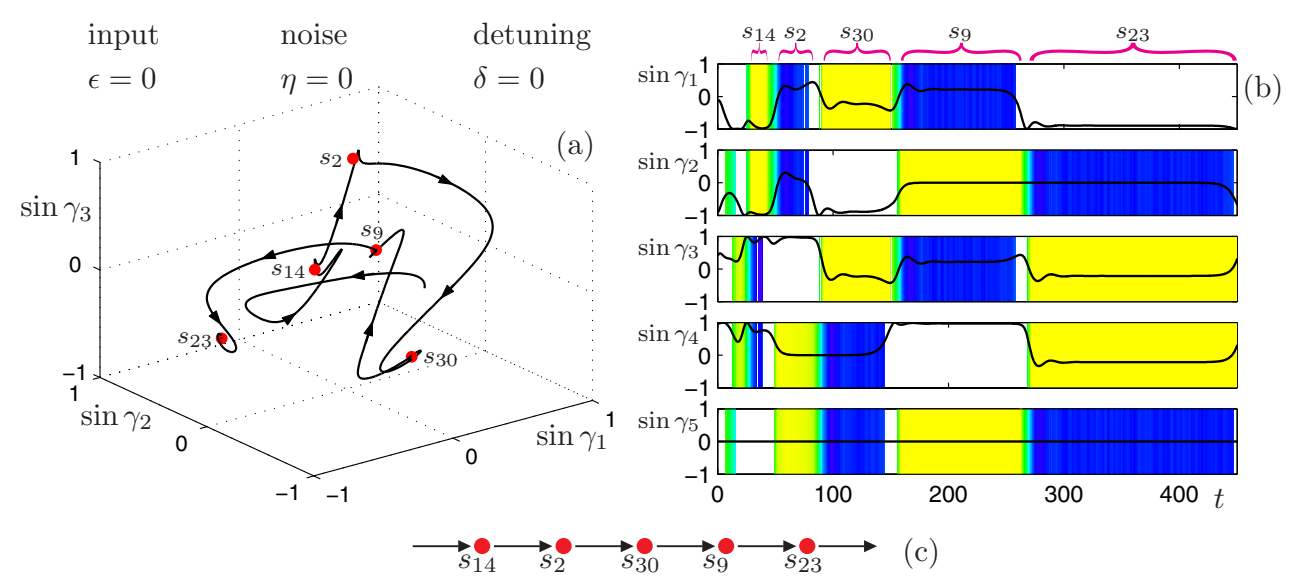

Figure 8. Approaching the heteroclinic network from an arbitrary initial condition in the unperturbed case (as labeled on the top left) for parameters (3.1). Panel (a) shows the dynamics in phase space: The labeled red dots show the three-cluster states approached by the trajectory that "travels close to" the heteroclinic connections. In panel (b) the time profiles are displayed: Plateaux of $\sin \gamma_{n}$ correspond to three-cluster states and transitions between them correspond to heteroclinic connections. Lining up the colored regions vertically reveals which oscillators form the stable yellow and unstable blue clusters, and the labels on the top identify the three-cluster states according to Table 1. Transition regions are colored light green. Panel (c) shows the path followed on the graph in Figure 6(b). See also the accompanying animation (68396_01.avi [3.9MB]).

which corresponds to the strength of attraction of the heteroclinic connections [18]. Notice in Figure 8(b) that the limit (3.15) is almost achieved even for small values $k=3,4,5$. Figure 8(c) shows the path followed on the graph in Figure 6(b) by the above dynamics.

The heteroclinic behavior changes when external perturbations such as noise $(\eta \neq 0)$, detuning $(\delta \neq 0)$, or impulsive inputs $(\epsilon \neq 0)$ are applied; see $(2.1)-(2.5)$. Figure 9 shows the response of the system to such perturbations; i.e., the effects of noise, detuning, and periodic impulsive inputs are shown in panels (a), (b), and (c), respectively, where exactly the same notation is used as in Figure 8. The precise dynamics followed depends on the initial condition, although after an initial transient the dynamics enters a neighborhood of the heteroclinic attractor. To avoid transient effects the initial condition is set close to the

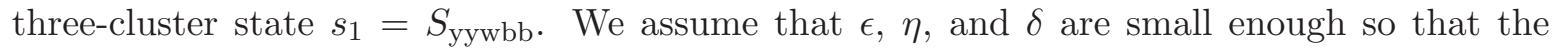
only attractors of the system are in a neighborhood of the heteroclinic attractor for the unperturbed case $\epsilon=\eta=\delta=0$. When the system is close to a three-cluster state it is sensitive to perturbations; that is, the perturbations determine which oscillator in the unstable blue cluster is advanced with respect to the other and, consequently, which of the two outgoing heteroclinic connections should be followed. The asymptotic stability of the network ensures that, after a transient, trajectories remain close to the heteroclinic network. This means that we can describe the dynamics by the itinerary of the heteroclinic network in Figure 6(a) (or the corresponding graph in Figure 6(b)). According to this we use the expressions "at a three-cluster state" and "at a node" to mean that the system is close to a three-cluster state. Furthermore, for the perturbed system the formula (3.15) does not hold. Instead, a characteristic time of transition between states can be assigned as shown by the length of plateaux in Figure 9(a2), (b2), (c2). This time is inversely proportional to the logarithm of 

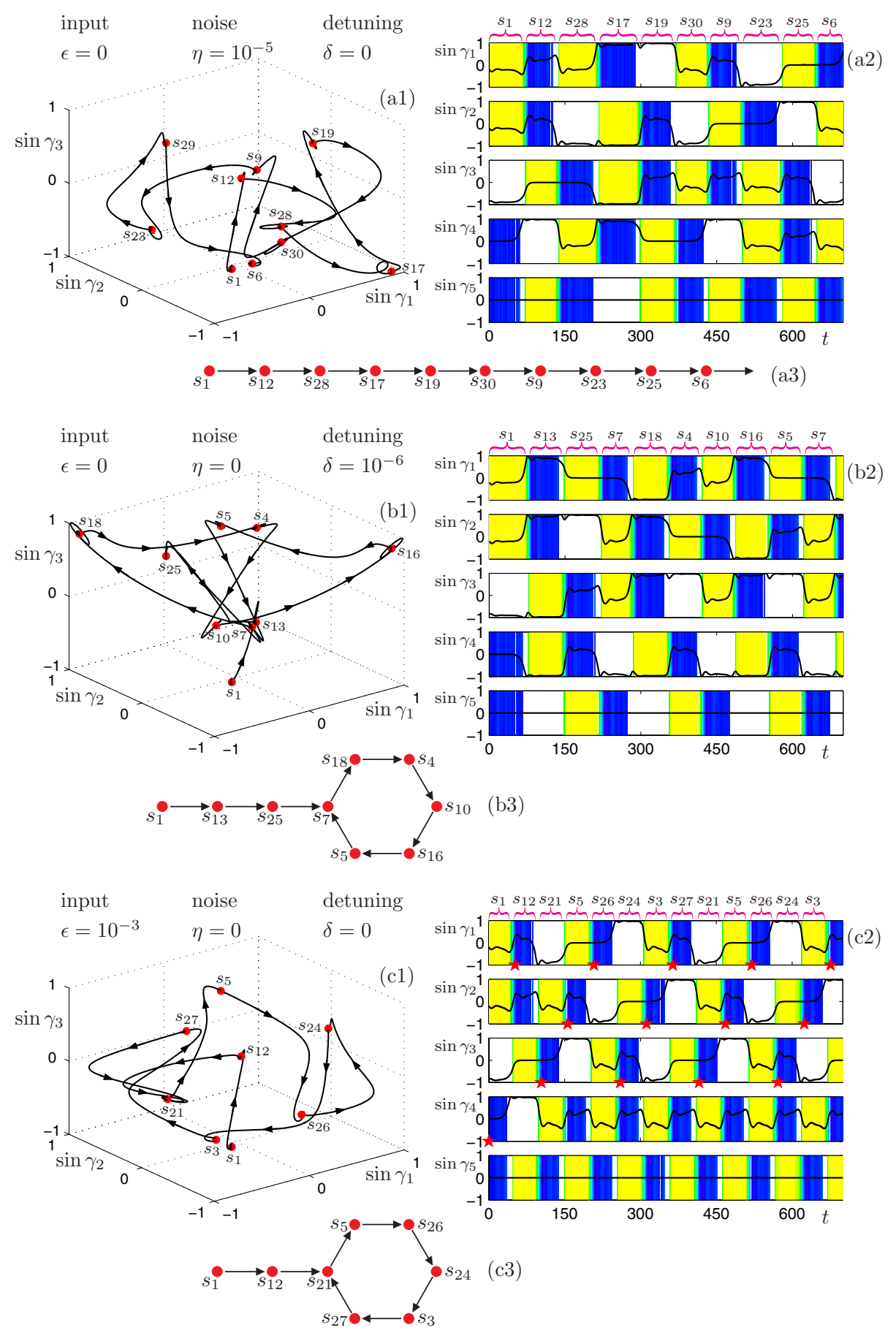

Figure 9. Dynamics of the system in the presence of noise (a), detuning (b), and periodic impulsive inputs (c) for parameters (3.1). Panels (a1), (b1), and (c1) show the dynamics in phase space; panels (a2), (b2), and (c2) depict the time profiles; and panels (a3), (b3), and (c3) show the paths followed in Figure 6(b). The same notation is used as in Figure 8. Panel (a2) corresponds to the raster plot in Figure 1. In panel (c2) red stars in every $t=k T=k 52.0$ indicate which oscillator receives the impulsive input. See also the accompanying animations (68396_02.avi [6.4MB]), (68396_03.avi [6.4MB]), and (68396_04.avi [6.7MB]). 
the magnitude of perturbations; see section 4.

In Figure 9(a) noise of strength $\eta=10^{-5}$ forces the system to follow a randomly chosen path on the heteroclinic network. At each three-cluster state/node the noise randomly chooses which of the two oscillators in the unstable blue cluster is advanced with respect to the other and, consequently, which of the two outgoing heteroclinic connections/directed edges is followed. Note that the whole network is explored with probability 1 if the simulation is run for long enough. The time domain $t \in[0,350]$ in Figure $9(\mathrm{a} 2)$ corresponds to the raster plot in Figure 1, where vertical lines indicate when the individual phases $\theta_{n}(t)$ go through zero.

Figure 9(b) shows the dynamics when oscillators are detuned according to the uniform detuning (2.5), which for $N=5$ means

$$
\omega_{1}=\omega-2 \delta, \quad \omega_{2}=\omega-\delta, \quad \omega_{3}=\omega, \quad \omega_{4}=\omega+\delta, \quad \omega_{5}=\omega+2 \delta .
$$

Here the detuning magnitude $\delta=10^{-6}$ is considered. Since the heteroclinic attractor is only robust to perturbations that preserve the symmetries of the system, it is generically destroyed by this detuning. More precisely, at a three-cluster state/node the detuning (3.16) always advances the blue oscillator with larger index (the one which changes its color to white in Figure 9(b2)). Consequently, one particular outgoing heteroclinic connection/directed edge is followed. This results (after a transient path) in the system reaching a cyclic path of length 6 (which is followed as $t \rightarrow \infty$ ). This path corresponds to limit cycle oscillations of the phase differences in the detuned system. Note that this limit cycle is not unique; i.e., for different initial states we can see different attractors, as discussed in section 4.2.

In Figure 9(c) the system is forced by periodic impulsive inputs of the form

$$
I_{i}(t)=\sum_{k=0}^{\infty} \delta(t-k T) \delta_{i p_{k}},
$$

where $\delta(t-k T)$ is a Dirac delta function while $\delta_{i p_{k}}$ is a Kronecker delta. The $k$ th term of this sum represents a unit impulse applied to the $p_{k}$ th oscillator at time $k T$, where $p_{k} \in$ $\{0,1, \ldots, 5\}\left(p_{k}=0\right.$ corresponds to no input). That is, the phase of the oscillator receiving the input is simply advanced by the input amplitude $\epsilon$. Here the input amplitude $\epsilon=10^{-3}$ and period $T=52.0$ are considered, and a red star is plotted in Figure $9(\mathrm{c} 2)$ when an oscillator receives an input. At a three-cluster state/node $s_{i}=S_{i_{1}, \ldots, i_{5}}$ at time $t=k T$ we can choose which of the two oscillators in the unstable blue cluster should receive the input (that is, $i_{p_{k}}=\mathrm{b}$ can be chosen) and, consequently, which of the two outgoing heteroclinic connections/directed edges should be followed. In Figure 9(c2) we always choose the blue oscillator with the smaller index to have its phase advanced (the blue oscillator with the red star changes its color to white). This results (after following the transient path) in a cyclic path of length 6 (which is followed as $t \rightarrow \infty$ ). Note that different input configurations lead to different paths and that repeated input sequences correspond to cyclic paths. This shows that by exploiting the natural dynamics of the system we can drive it along a chosen path on the heteroclinic network: we can think of the dynamics as an input-output system which is capable of performing finite-state computation [3]. The reliability of such computations is investigated in detail in the next section. 


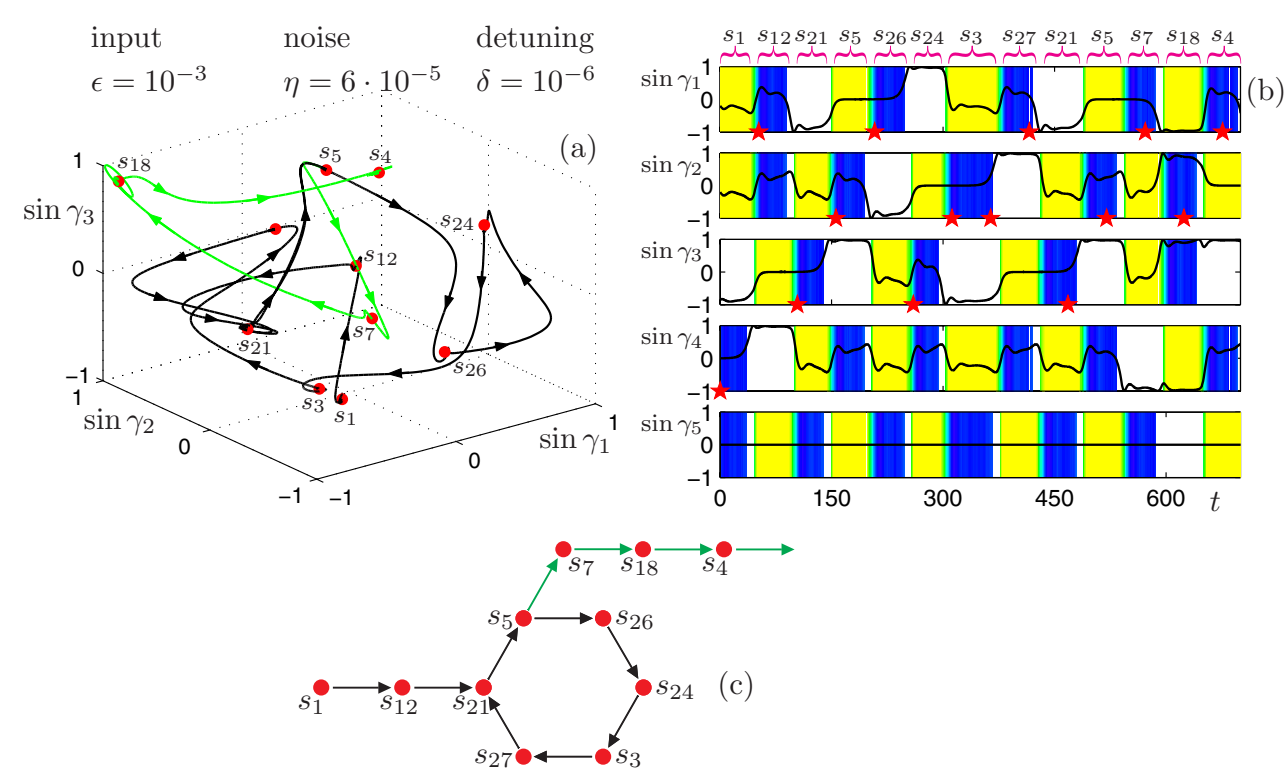

Figure 10. Dynamics of the system in the presence of periodic impulsive inputs, noise, and detuning (as labeled on the top left) for parameters (3.1). The same notation is used as in Figure 8. In panel (b) red stars in every $t=k T=k 52.0$ indicate which oscillator receives the impulsive input. In panels (a) and (c) the light green sections of the trajectory and the graph show the dynamics after an incorrect switch at the three-cluster state $s_{5}=S_{\text {ybwby }}$.

4. Reliability of switching along the heteroclinic network. In this section we illustrate that it is possible to reliably drive the system around the heteroclinic network of three-cluster states using small impulsive inputs. This can be managed even when noise and detuning are present if the input amplitude is large enough relative to the noise strength and detuning magnitude. From now on we will use the term switch to mean a transition along a heteroclinic orbit between three-cluster states.

For example, one may check that by applying the same noise as in Figure 9(a) (with strength $\eta=10^{-5}$ ), the same detuning as in Figure 9(b) (with magnitude $\delta=10^{-6}$ ), and the same input as in Figure 9(c) (with amplitude $\epsilon=10^{-3}$ and period $T=52.0$ ), the obtained trajectory, time profiles, and, consequently, the path followed are indistinguishable from those in Figure 9(c), where neither noise nor detuning are present. This means that the dynamics is still determined by the sequence of inputs.

Figure 10 shows the response of the system to perturbations with noise increased to the extent that the switches are not all determined by the inputs. (The same notation is used as in Figure 8 and the initial condition is at the three-cluster state $s_{1}=S_{\text {yywbb.) Again the same }}$ detuning is applied as in Figure 9(b) (with magnitude $\delta=10^{-6}$ ), the same input is applied as in Figure 9(c) (with amplitude $\epsilon=10^{-3}$ and period $T=52.0$ ), but the noise (of strength $\eta=6 \cdot 10^{-5}$ ) is larger than in Figure $9(\mathrm{a})$. The trajectory and the time profiles are initially close to those in Figure 9(a) and (b).

Figure 10(b) shows that the first "incorrect" switch happens at time $t=6 T=312.0$, where the first blue oscillator receives an impulsive input but the system stays at the threecluster state $s_{3}=S_{\text {ybwyb }}$ instead of switching to $s_{27}=S_{\text {bwyby }}$. Another impulsive input has 
to be applied to the same oscillator at time $t=7 T=364.0$ in order to force the system to switch. The next "incorrect" switch takes place at time $t=10 T=520.0$, where an impulsive input is applied to the first blue oscillator of the three-cluster state $s_{5}=S_{\text {ybwby }}$; that is, a switch to $s_{26}=S_{\text {ybwby }}$ is initiated. Instead, a switch to $s_{7}=S_{\text {byywb }}$ is observed (the blue oscillator without a red star changes its color to white; i.e., the "wrong" heteroclinic connection/directed edge is followed). This "incorrect" switch leads the system away from the cyclic path of length 6, as shown by the green part of the trajectory in Figure 10(a) and by the green part of the graph in Figure 10(c).

In order to measure the accuracy of switching, at each three-cluster state we randomly choose which blue oscillator receives the impulsive input (i.e., choose which heteroclinic connection/directed edge should be followed), and then detect whether the expected switch happens. To be more precise, we classify the system as being at a three-cluster state $s_{i}$ if $\theta(t) \in B_{\xi}\left(s_{i}\right)$, where $B_{\xi}\left(s_{i}\right)$ denotes the $\xi$-neighborhood of $s_{i}$. The detection tolerance $\xi>0$ is chosen such that the $\xi$-neighborhoods of different three-cluster states do not intersect each other, that is, $B_{\xi}\left(s_{i}\right) \cap B_{\xi}\left(s_{j}\right)=\emptyset$ if $i \neq j$. In this article we fix $\xi=0.5$, but no qualitative change is observed when using different $\xi$.

Suppose that we are at the three-cluster state $s_{i}=S_{i_{1}, \ldots, i_{5}}$ at time $t=k T$ and randomly choose an oscillator $p_{k}$ in the unstable blue cluster for input (that is, $i_{p_{k}}=\mathrm{b}$ ). Then we predict $s_{j}=O_{p_{k}}\left(s_{i}\right)$ to be the state we expect after a time $T$; see definitions (3.13), (3.14) and Table 2. We declare a switch to be correct if $\theta(t+T)$ is at the expected three-cluster state $s_{j}$ and otherwise incorrect. An incorrect switch can occur for one of the three reasons:

(i) we cannot classify the state of $\theta(t)$ (i.e., there is no $i$ such that $\theta(t) \in B_{\xi}\left(s_{i}\right)$ ),

(ii) we cannot classify the state of $\theta(t+T)$, or

(iii) we can classify both states but the switch is not as expected (i.e., $O_{p_{k}}\left(s_{i}\right) \neq s_{j}$ ), as can be observed in Figure 10(b).

Figure 11 shows 14 randomly chosen switches for perturbations $\epsilon=10^{-3}, \eta=10^{-7}$, and $\delta=0$ with $T=85.0$. (Again the same notation is used as in Figure 8 and the initial condition is the three-cluster state $s_{1}=S_{\text {yywbb. }}$ ) The system follows the randomly chosen path expected from the provided sequence of impulsive inputs; that is, all switches are correct (the blue oscillators with red stars change their color to white in Figure 11(a), and the sequence of three-cluster states can be predicted by $\left.s_{j}=O_{p_{k}}\left(s_{i}\right)\right)$. In the next section we compute the rate of incorrect switches as a function of input period $T$, input amplitude $\epsilon$, noise strength $\eta$, and detuning magnitude $\delta$.

4.1. Error rates for switching. In order to check how reliable the switches are when driving the system with impulsive inputs in the presence of noise and detuning, we perform long simulations and count the proportion of correct and incorrect switches. The error rate $0 \leq E \leq 1$ is calculated as the proportion of incorrect switches for a large number of attempts (2000 switches) for a random choice of the possible switches at each state. The random choice is uniformly chosen between the two possible inputs that initiate switching at each step. More precisely, if we are at the three-cluster state $s_{i}=S_{i_{1}, \ldots, i_{5}}$, we choose with equal probability one of the two $p_{k}$ such that $i_{p_{k}}=\mathrm{b}$; that is, an oscillator in the unstable blue cluster is perturbed. If no three cluster-state $s_{i}$ is identified we choose $p_{k}=0$; i.e., no input is provided. The error rate clearly depends on the exact values of the parameters in (2.2), which here are fixed as in (3.1). It also depends on the input period $T$, the input amplitude $\epsilon$, the noise strength $\eta$, 


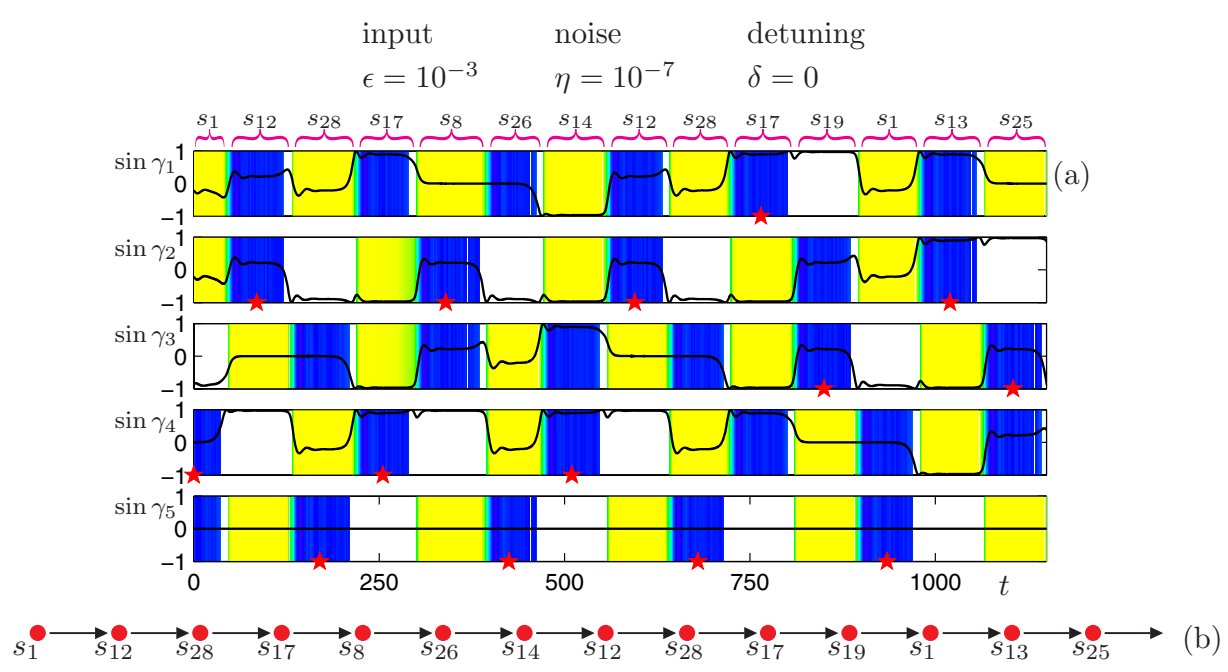

Figure 11. Driving the system along a randomly chosen path in the heteroclinic network for parameters (3.1) with perturbations labeled on the top. The same notation is used as in Figure 8. In panels (a) and (b) the time profiles and the followed path are shown for the 14 switches. In panel (a) red stars in every $t=k T=k 85.0$ indicate which oscillator receives the impulsive input. See also the accompanying animation (68396_05.avi $[10.2 \mathrm{MB}])$.

and the detuning magnitude $\delta$; that is, $E=E(T, \epsilon, \eta, \delta)$. We will assume that the error rate converges for our assumptions on random choice of $\left\{p_{k}\right\}$.

One can maintain a periodic orbit near the heteroclinic network with period $T>T_{\epsilon}$ between successive states by application of impulsive inputs of amplitude $\epsilon$, where

$$
T_{\epsilon}=-\frac{1}{\lambda_{e}} \ln \epsilon+\mathcal{O}(1)=-\frac{\ln (10)}{\lambda_{e}} \log _{10} \epsilon+\mathcal{O}(1),
$$

as $\epsilon \rightarrow 0$. Here $\lambda_{e}$ is the expanding eigenvalue of the three-cluster states (for parameters (3.1) we have $\lambda_{e}=\lambda_{3}=0.1703$, as given by (3.8) and shown in Figure 4(a)). Scaling (4.1) can be seen by the following argument. Consider a transition from the three-cluster state $s_{i}$ to the three-cluster state $s_{j}$ with symmetrically related surfaces of section $\Sigma_{i}$ and $\Sigma_{j}$, intersecting the stable manifolds of $s_{i}$ and $s_{j}$, respectively. If we provide an impulse of amplitude $\epsilon$ in the unstable direction at $\Sigma_{i}$, this will grow proportionally to $\epsilon \mathrm{e}^{\lambda_{e} t}$, meaning that it saturates and causes a switch when $\epsilon \mathrm{e}^{\lambda_{e} T}=\mathcal{O}(1)$. This in turn implies (4.1). If we perturb a three-cluster state $s_{i}$ in its unstable direction, then the unstable direction for $s_{j}$ is not affected, meaning that by choosing to perturb on a section $\Sigma_{i}$ closer to $s_{i}$, we can provide impulses of amplitude $\epsilon$ to obtain periodic orbits with any period $T>T_{\epsilon}$.

Similarly, it can be shown [24] that the addition of noise of strength $\eta$ will transform the heteroclinic attractor into an approximately periodic attractor with period $T \approx T_{\eta}$, where

$$
T_{\eta}=-\frac{1}{\lambda_{e}} \ln \eta+\mathcal{O}(1)=-\frac{\ln (10)}{\lambda_{e}} \log _{10} \eta+\mathcal{O}(1),
$$

as $\eta \rightarrow 0$. In consequence, we conclude that one can navigate around the network with error 

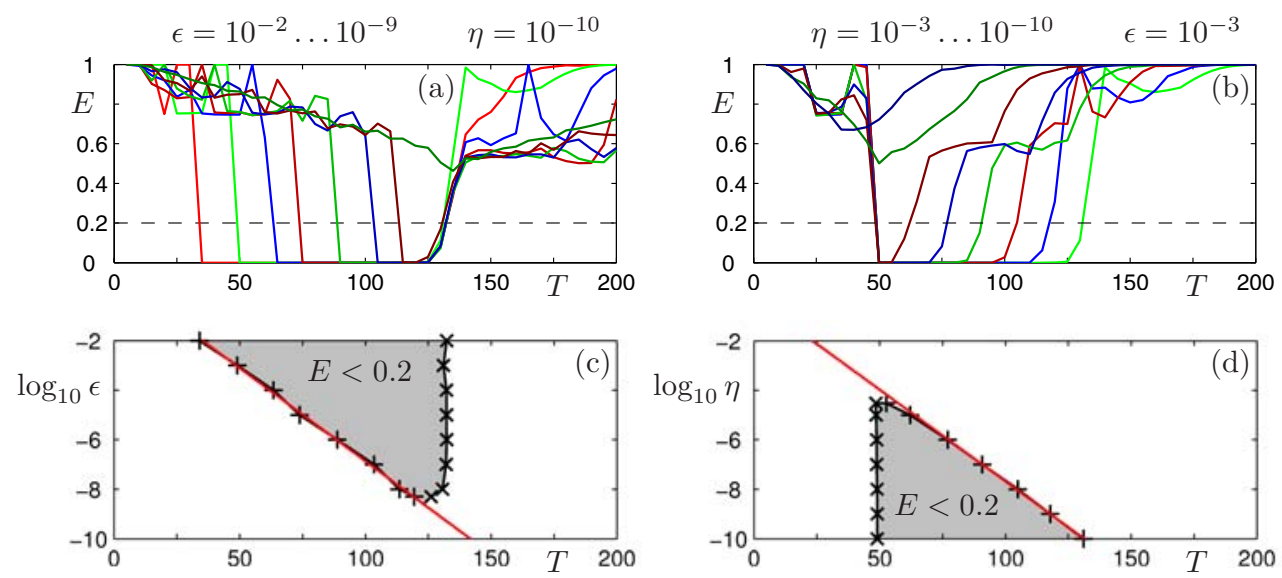

Figure 12. In panels (a) and (b) the error rate $E$ is shown as a function of the input period $T$ for parameters (3.1). In panel (a) several different values of the input amplitude $\epsilon$ are considered for fixed noise strength $\eta$, while in panel (b) several different values of $\eta$ are taken for fixed $\epsilon$. No detuning is added $(\delta=0)$. The leftmost curves in panels (a) and (b) correspond to the largest values of $\epsilon$ and $\eta$, respectively. In panels (c) and (d) the shaded domains show where the error rate is below the threshold 0.2 in the $\left(T, \log _{10} \epsilon\right)-p l a n e$ and in the $\left(T, \log _{10} \eta\right)$-plane, respectively. Notice that the fitted red lines have approximately the same gradient in both panels (c) and $(\mathrm{d})$.

rate $E$ close to zero as long as $T$ is in the range

$$
T_{\epsilon}<T<T_{\eta}
$$

If $T<T_{\epsilon}$, then errors take place because the time is not long enough to permit the connections to be followed. If $T>T_{\eta}$, then the noise will be the dominant influence in determining when transitions take place, and hence the error rate will be large. Note that a range of possible $T$ in (4.3) is available as long as

$$
\epsilon \gg \eta
$$

If both noise $\eta>0$ and detuning $\delta>0$ are present in the system, then the same argument holds except one must write $\max (\delta, \eta)$ in place of $\eta$ in formulae (4.2)-(4.4).

In Figure 12(a) and (b) the error rates (obtained from 2000 switches) are shown as a function of the input period $T$ for several different values of input amplitude $\epsilon$ and noise strength $\eta$, respectively (with no detuning, $\delta=0$ ). The leftmost curves in Figure 12(a) and (b) correspond to the largest values of $\epsilon$ and $\eta$, respectively. These panels clearly show that when (4.4) is satisfied there exists an interval of $T$ (4.3) where $E \approx 0$. Also notice that left and right boundaries of this interval move proportionally to $\log _{10} \epsilon$ in Figure 12 (a) and proportionally to $\log _{10} \eta$ in Figure 12(b). In order to measure these boundaries more precisely we detect where the curves intersect the error rate $E=0.2$ (denoted by horizontal dashed lines in Figure 12(a) and (b)). We plot the corresponding data in Figure 12(c) and (d), where the low error rate domains $(E<0.2)$ are shaded. The least square fitted red lines in Figure $12(\mathrm{c})$ and (d) with gradients

$$
g_{\epsilon}=-0.0749 \pm 0.0014 \quad \text { and } \quad g_{\eta}=-0.0740 \pm 0.0005,
$$



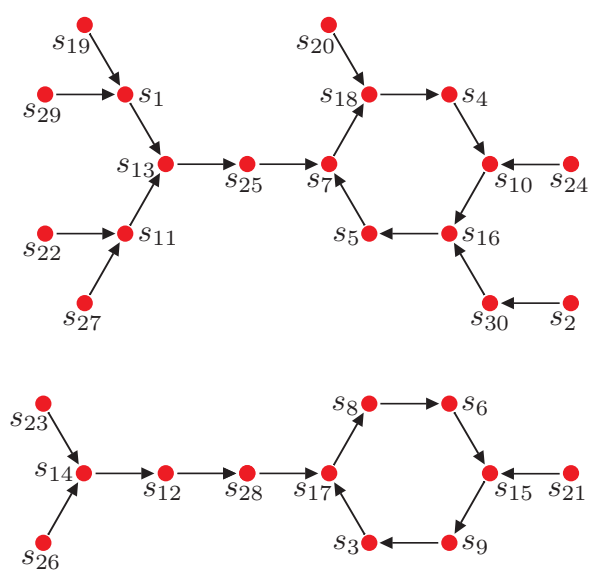

Figure 13. The network in Figure 6(b) collapses to this network with two connected components on applying the uniform detuning (3.16). Observe that there are two eventually periodic paths of length 6 , indicating that detuning results (after a transient path) in one of two "periodic spatiotemporal codes" depending on initial state.

respectively, agree well with the predicted gradients in (4.1) and (4.2), namely,

$$
g=-\frac{\ln (10)}{\lambda_{e}}=-\frac{\ln (10)}{\lambda_{3}}=-0.0740
$$

This shows that the accuracy of navigation around the heteroclinic network can be predicted by the stability properties of the three-cluster states.

4.2. Detuning-driven spatiotemporal coding. In addition to giving a network that can perform finite-state computations, the system (2.1), (2.2) can give spatiotemporal coding of steady external inputs that are in the form of detuning. Assuming the uniform detuning (3.16) and setting the initial condition at $s_{1}=S_{\text {yywbb }}$ the system reaches a cyclic path of length 6 (after some transient path) as shown in Figure 9(b). In fact, this path encodes information about the form of the detuning, as discussed below.

Here we assume the uniform detuning (3.16) and consider the system starting at different three-cluster states. Whenever the system is at a three-cluster state $s_{i}=S_{i_{1}, \ldots, i_{5}}$ there will be a "preferred" direction along the graph in Figure 6(b). Namely, if $i_{p}=i_{q}=\mathrm{b}$ are the unstable blue oscillators, then if $\omega_{i_{p}}>\omega_{i_{q}}$ the detuning will tend to initiate a switch to $O_{i_{p}}\left(s_{i}\right)$, while if $\omega_{i_{p}}<\omega_{i_{q}}$ the initiated transition will be to $O_{i_{q}}\left(s_{i}\right)$; see definitions (3.13), (3.14) and Table 2. Hence, uniform detuning causes a bias to the switchings, meaning that one of the outgoing edges is removed from each node and the network with the graph in Figure 13 remains for the original network. This "detuned" graph consists of two connected components, each of which has an absorbing cyclic path of length 6 and transient paths that are asymptotic to these circuits after a finite number of switches. Which of the cyclic paths is reached depends on the initial state. The sequences of three-cluster states along the circuits can therefore be considered as spatiotemporal encodings of the applied detuning.

Notice that the magnitude of $\delta$ does not change the graph in Figure 13, but it does affect the average period between switches $T_{\delta}$ in a similar way to (4.1). Different detuning configurations obtained by permutation of the indices in (3.16) result in different cyclic paths 

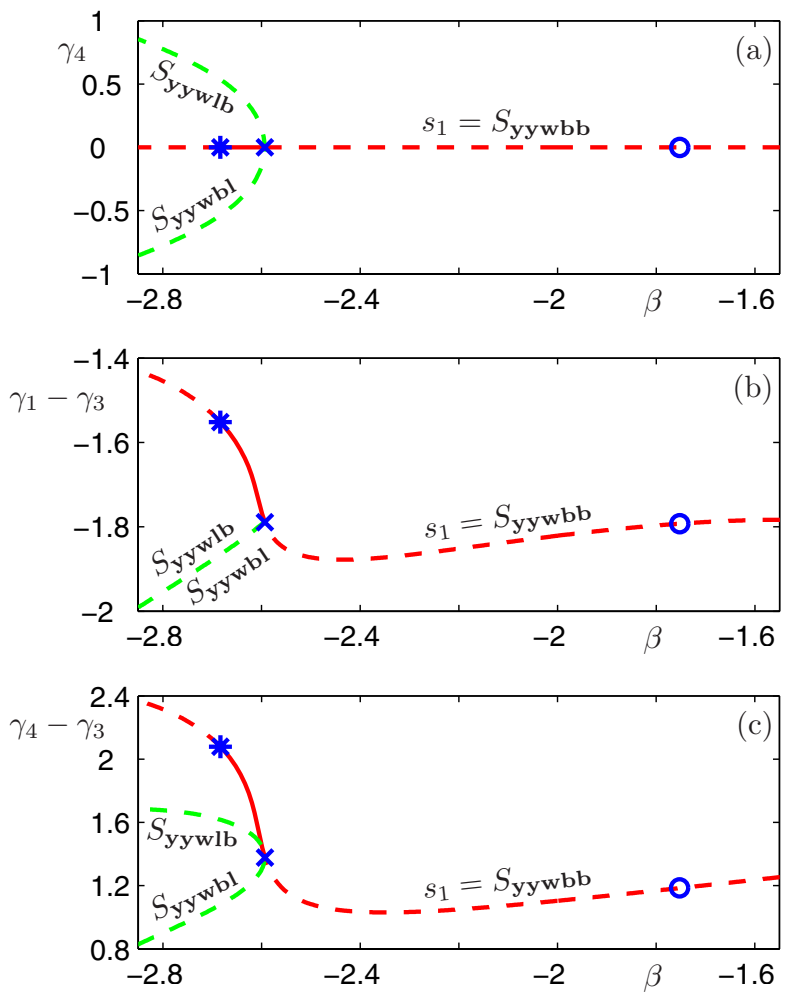

Figure 14. Bifurcation diagrams showing the phase differences $\gamma_{4}=\theta_{5}-\theta_{4}$ (a), $\gamma_{1}-\gamma_{3}=\theta_{1}-\theta_{3}$ (b), and $\gamma_{4}-\gamma_{3}=\theta_{4}-\theta_{3}$ (c) as the parameter $\beta$ is varied for $r=0.2, \alpha=1.8, \omega=1$ with $\epsilon=\eta=\delta=0$. The three-cluster state $s_{1}=S_{\mathrm{yywbb}}$ and the bifurcated cluster states $S_{\mathrm{yywlb}}, S_{\mathrm{yywbl}}$ are labeled on the corresponding red and light green branches. Solid curves denote stable states and dashed curves denote saddle states. The blue star at $\beta=-2.6840$, blue cross at $\beta=-2.5933$, and blue circle at $\beta=-1.7515$ denote the Hopf, pitchfork, and resonance bifurcations, respectively. The network shown in Figure 6 is attracting between the pitchfork and the resonance bifurcations.

of length 6 , that is, different spatiotemporal codes. One may check that 120 cyclic paths of length 6 exist (using the adjacency matrix defined in section 3.2 we obtain $\operatorname{tr}\left(A^{6}\right)=120$ ). However, only 20 of these paths are possible absorbing cycles when one considers all possible $5 !=120$ permutations of $(3.16)$.

5. Excitable dynamics. The dynamics described in sections 3 and 4 is robust (structurally stable); i.e., there is an open set of parameters around (3.1) where such an attracting heteroclinic network exists. We observe the network on varying $\beta$ in the range $(-2.5933,-1.7515)$ and now analyze the bifurcations at the boundaries of this regime. At the lower boundary the saddles within the network are stabilized at a pitchfork bifurcation to become stable threecluster states that remain arranged in a network as in Figure 6. At the upper boundary the heteroclinic network persists but loses stability at a resonance bifurcation [9].

As verification of this, Figure 14 shows bifurcation diagrams for the three-cluster state $s_{1}=S_{\text {yywbb }}$. The parameters $r, \alpha$, and $\omega$ are given by (3.1) except that $\beta$ is varied, and we set $\epsilon=\eta=\delta=0$. The figure was generated by the continuation package AUTO [10].

Copyright $@$ by SIAM. Unauthorized reproduction of this article is prohibited. 

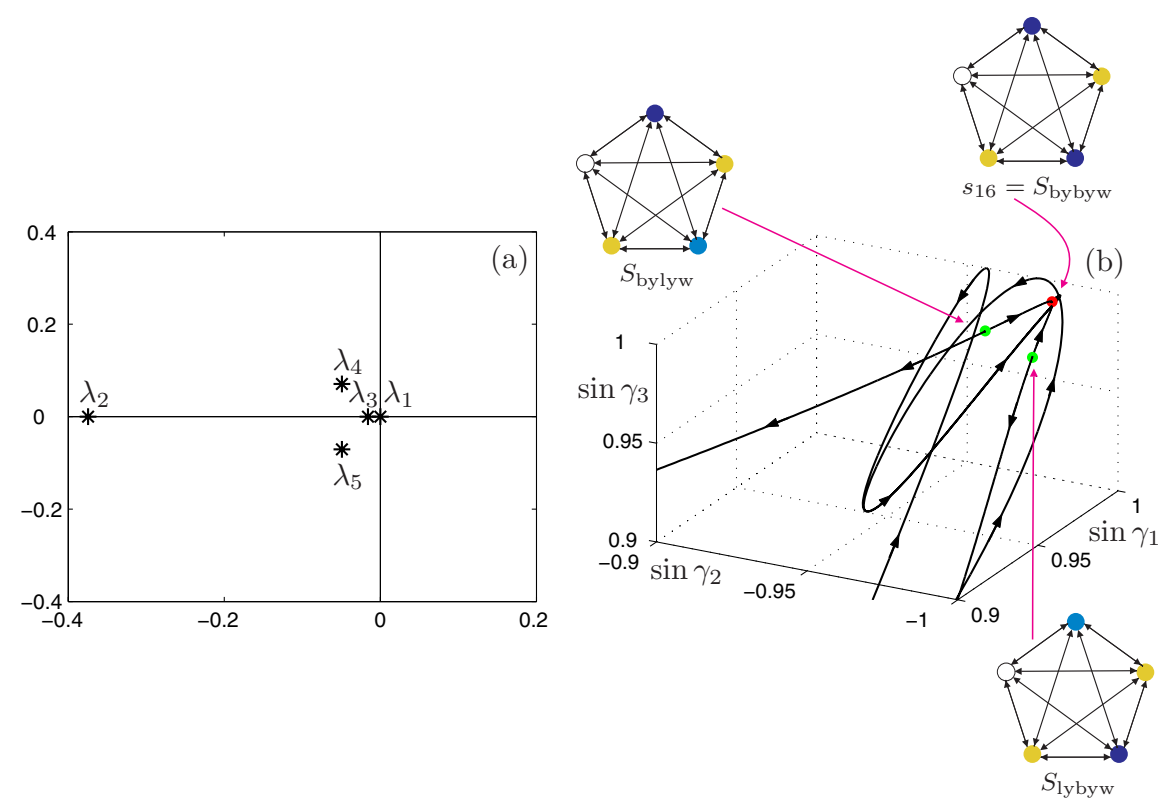

Figure 15. Dynamics around a stable three-cluster state for parameters (5.1) in the unperturbed case $\epsilon=\eta=\delta=0$. In panel (a) the eigenvalues (3.7), (5.3) are displayed in the complex plane. Panel (b) shows the local dynamics in phase space in the vicinity of the three-cluster state $s_{16}=S_{\text {bybyw }}$ (red dot). Light green dots show the bifurcated saddle cluster states $S_{\mathrm{lybyw}}$ and $S_{\mathrm{bylyw}}$. One branch of the unstable manifold of each of these states connects to $s_{16}=S_{\text {bybyw }}$.

In Figure 14 the phase differences are plotted as a function of the parameter $\beta$. Solid lines correspond to stable states, and dashed lines correspond to saddle states. Blue star, blue cross, and blue circle represent Hopf, pitchfork, and resonance bifurcations, respectively, from the three-cluster state. Between the pitchfork and the resonance bifurcation the heteroclinic network is attracting because $\left|\lambda_{2}\right|>\lambda_{3}$ (as in Figure 4(a)); that is, the contracting eigenvalue is stronger than the expanding one for the robust connection. The pitchfork bifurcation occurs when $\lambda_{3}=0(\beta=-2.5933)$, while the resonance occurs when $\left|\lambda_{2}\right|=\lambda_{3}(\beta=-1.7515)$.

Figure 14(a) shows the phase difference $\gamma_{4}=\theta_{5}-\theta_{4}$ as a function of $\beta$. For the saddle three-cluster state $s_{1}=S_{\text {yywbb }}$ (horizontal red line) the phase difference $\gamma_{4}=\theta_{4}-\theta_{5}$ is zero, indicating that the 4 th and the 5 th oscillators are synchronized. At the pitchfork bifurcation (blue cross at $\beta=-2.5933$ ) the three-cluster state $s_{1}=S_{\text {yywbb }}$ becomes stable with $\operatorname{Re}\left(\lambda_{n}\right)<0$ for $n=2,3,4,5$ (see Figure 15(a)). The bifurcation creates two branches of saddle cluster states (light green curves) where $\gamma_{4} \neq 0$, i.e., the 4 th and the 5 th oscillators are not synchronized. If $\gamma_{4}>0$, for this cluster state we write $S_{\text {yywlb }}$ (and $S_{\text {yywbl }}$ if $\gamma_{4}<0$ ). The subscript "l" stands for the light blue color used in Figures 15-16 and indicates the oscillator in the unstable blue cluster whose phase has been advanced. These new cluster states are located in the subspace (3.11), and their unstable manifolds are connected to the three-cluster states $s_{1}=S_{\text {yywbb }}, s_{12}=S_{\text {bbywy }}$, and $s_{13}=S_{\text {bbyyw }}$, as detailed further below.

Figure 14(b) and (c) show the phase differences $\gamma_{1}-\gamma_{3}=\theta_{1}-\theta_{3}$ and $\gamma_{4}-\gamma_{3}=\theta_{4}-\theta_{3}$ as a function of $\beta$ for the relevant cluster states. For $s_{1}=S_{\text {yywbb }}$ (red curve) we have $\gamma_{1}-\gamma_{3}=\chi$ and $\gamma_{4}-\gamma_{3}=\psi$; see definitions (3.3), (3.4). Notice that in Figure 14(b) the branches of 


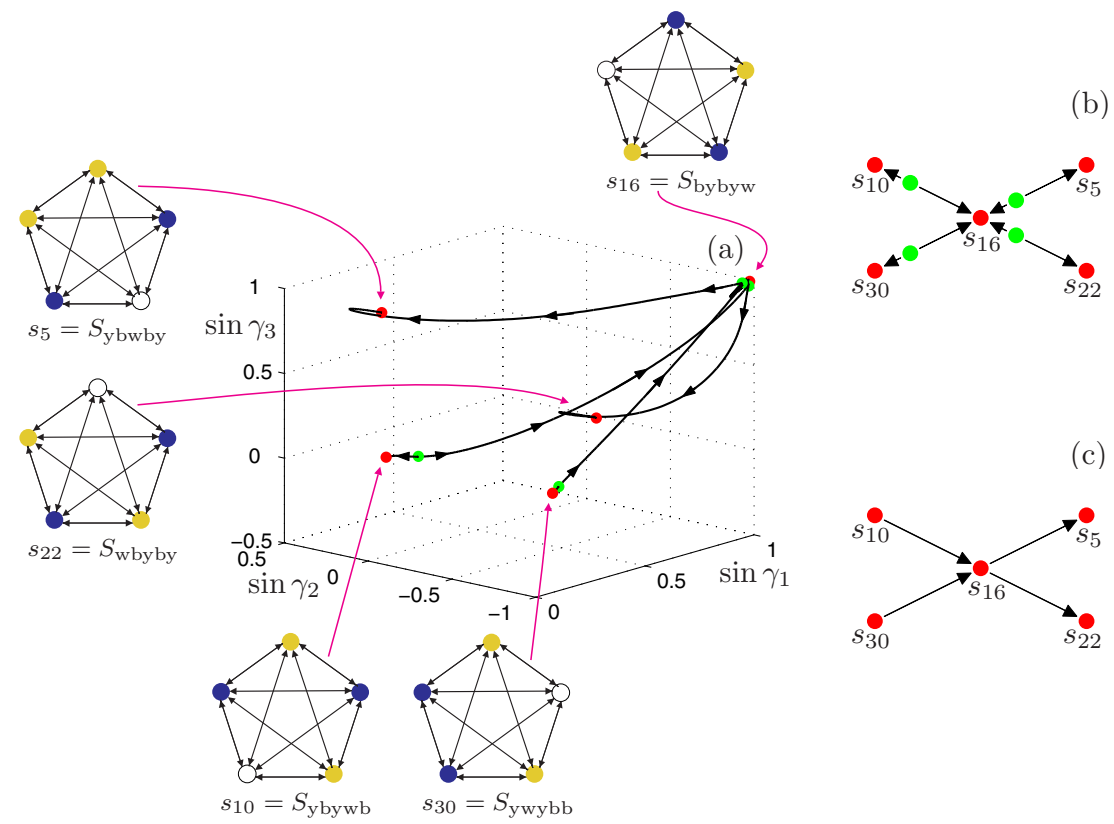

Figure 16. In panel (a) the heteroclinic connections originated in and terminated at the three-cluster state $s_{16}=S_{\text {bybyw }}$ are shown in phase space for parameters (5.1) in the unperturbed case $\epsilon=\eta=\delta=0$. The cluster states are represented by red and light green dots. In panel (b) a simple graph representation is displayed such that the nodes and directed edges correspond to cluster states and heteroclinic connections, respectively. Panel (c) shows a further simplified graph representation where only the three-cluster states are displayed and the directed edges represent possible large-input-triggered switches between them.

cluster states $S_{\text {yywlb }}$ and $S_{\text {yywbl }}$ overlap (light green curve), which indeed shows that these cluster states are embedded in the subspace (3.11). The symmetric copies of $s_{1}=S_{\mathrm{yywbb}}$, as in Table 1, behave in a corresponding way at the pitchfork bifurcation.

On reducing $\beta$ further, the complex conjugate pair of eigenvalues moves to the right-hand side of the imaginary axis at the Hopf bifurcation (blue star at $\beta=-2.6840$ ), and the threecluster state $s_{1}=S_{\text {yywbb }}$ becomes a saddle again since $\lambda_{2}, \lambda_{3}<0$ and $\operatorname{Re} \lambda_{4}=\operatorname{Re} \lambda_{5}>0$. However, the resulting dynamics is different from that on the right side of the pitchfork bifurcation (the oscillatory branches emerging at the Hopf bifurcation are not studied further in this paper).

At the resonance bifurcation (blue circle at $\beta=-1.7515)$ the contracting eigenvalue becomes weaker than the expanding eigenvalue, i.e., $\left|\lambda_{2}\right|<\lambda_{3}$. Consequently, the heteroclinic network becomes repelling; see [9]. Note that the three-cluster states persist as saddles and the network still persists on the right side of the resonance bifurcation. However, since it is not attracting, general initial conditions may approach long-period stable limit cycles or even chaotic attractors (see section 7).

5.1. Network of excitable three-cluster states. We now investigate in detail the network of stable excitable states that replaces the heteroclinic network to the left side of the pitchfork bifurcation, by considering the parameters

$$
r=0.2, \quad \alpha=1.8, \quad \beta=-2.6, \quad \omega=1.0 .
$$

Copyright (c) by SIAM. Unauthorized reproduction of this article is prohibited. 
Note that only $\beta$ has been changed compared to parameters (3.1). Solving (3.5) for parameters (5.1), one can find a three-cluster state (3.3), (3.4) with

$$
\chi=-1.7638, \quad \psi=1.4594, \quad \Omega=0.9343,
$$

and symmetric copies as in Table 1. Substituting (5.1), (5.2) into (3.7), the eigenvalues are

$$
\lambda_{2}=-0.3746, \quad \lambda_{3}=-0.0158, \quad \lambda_{4,5}=-0.0493 \pm \mathrm{i} 0.0709,
$$

as displayed in Figure 15(a). Notice that all eigenvalues are to the left of the imaginary axis indicating that the three-cluster states are stable, but $\lambda_{3}$ is close to zero as the system is close to the bifurcation point.

In Figure 15(b) the stable three-cluster state $s_{16}=S_{\text {bybyw }}$ (red dot) and the bifurcated saddle cluster states $S_{\text {lybyw }}$ and $S_{\text {bylyw }}$ (light green dots) are displayed (with the oscillators corresponding to the subscript "l" colored light blue). The latter cluster states are located in the symmetric copy of the subspace (3.11). Observe that now $s_{16}=S_{\text {bybyw }}$ is attracting from all directions. In particular, $\operatorname{Re}\left(v_{4}\right)=\operatorname{Re}\left(v_{5}\right)$ and $\operatorname{Im}\left(v_{4}\right)=-\operatorname{Im}\left(v_{5}\right)$ correspond to the spiralling directions, while $v_{3}$ and $-v_{3}$ are the directions toward the three-cluster states $S_{\text {lybyw }}$ and $S_{\text {bylyw }}$. (The latter two directions are indeed the opposite of each other, but the curvature of the projection $\left(\sin \gamma_{1}, \sin \gamma_{2}, \sin \gamma_{3}\right)$ is large in the vicinity of the three-cluster state $s_{16}=S_{\text {bybyw }}$ as it is close to $(1,-1,1)$.)

It can be verified numerically that one branch of the one-dimensional unstable manifold of the cluster state $S_{\text {lybyw }}$ returns to $s_{16}=S_{\text {bybyw }}$ while the other branch connects to the threecluster state $s_{22}=S_{\text {wbyby }}$. Similarly, the unstable manifold of $S_{\text {bylyw }}$ connects to $s_{16}=S_{\text {bybyw }}$ and $s_{5}=S_{\text {ybwby }}$; this is illustrated in Figure 16(a). There are saddle cluster states $S_{\text {ybywl }}$ and $S_{\text {ywybl near }} s_{10}=S_{\text {ybywb }}$ and $s_{30}=S_{\text {ywybb }}$, respectively. These are located in the symmetric copy of the subspace (3.12) and their unstable manifolds connect to $s_{16}=S_{\text {bybyw }}$. This means that there are connections as shown in the graph Figure 16(b), where again the dots represent cluster states and directed edges represent heteroclinic connections between them.

Hence, by providing a large enough perturbation when the system is near $s_{16}=S_{\text {bybyw }}$ in the direction of $S_{\text {lybyw }}$, it is possible to "jump over" the stable manifold of $S_{\text {lybyw }}$ and then approach $s_{22}=S_{\text {wbyby }}$. We say that the stable three-cluster states are excitable to large perturbations that separate the blue cluster. Taking these large perturbations into account we can obtain the simplified graph of connections in Figure 16(c). Notice that this graph is close to the one in Figure 5(b) for the original heteroclinic network with the difference that in Figure 5(b) infinitesimally small perturbations can trigger transitions while in Figure 16(c) the perturbations have to be of finite size.

Considering all three-cluster states the graph in Figure 6(b) is obtained again as a representation of the system when finite size perturbations are applied. We stress that in the resulting network of excitable three-cluster states, the excitability is a property of the threecluster states and not a property of the individual oscillators. We will use the term excitable network to describe this dynamics.

5.2. Reliable switching in the excitable network. We now demonstrate that it is possible to reliably navigate around the graph of three-cluster states by applying finite size impulsive inputs in precisely the same way as described in section 4. Figure 17(a) shows an example

Copyright $@$ by SIAM. Unauthorized reproduction of this article is prohibited. 


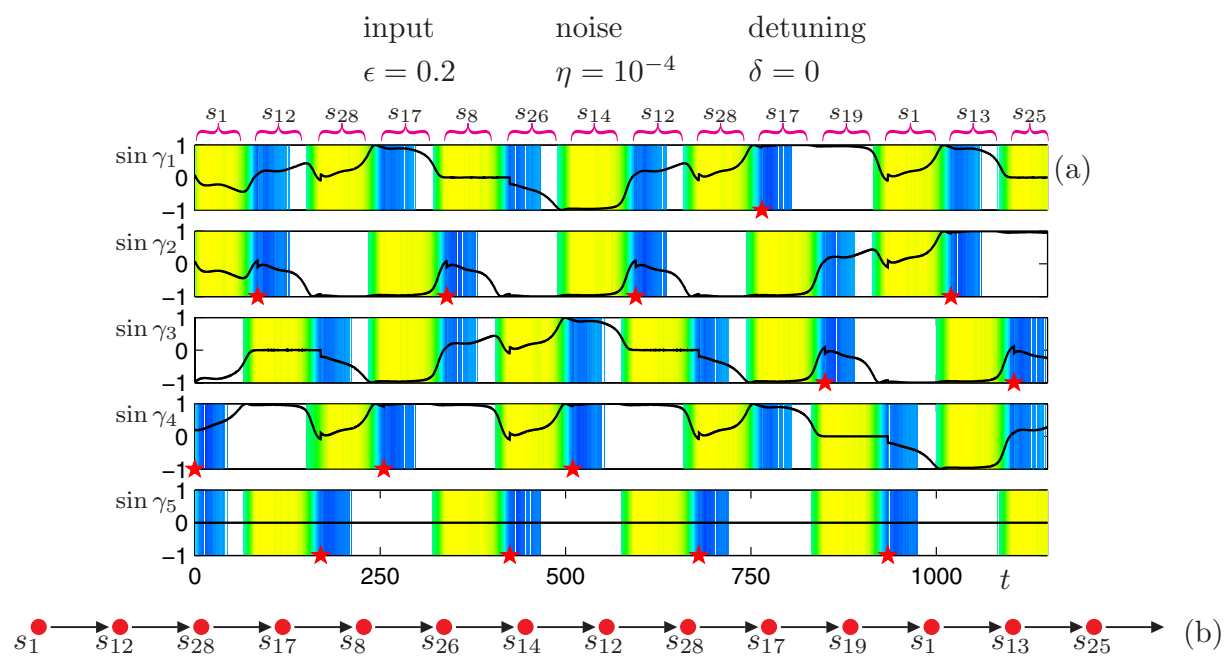

Figure 17. Driving the system along a randomly chosen path of the network of excitable three-cluster states for parameters (5.1) with perturbations labeled on the top. The same notation is used as in Figure 8 . In panels (a) and (b) the time profiles and the followed path are shown for the first 14 switches. In panel (a) red stars in every $t=k T=k 85.0$ indicate which oscillator receives the input. Notice that on providing the same sequence of inputs as in Figure 11 the same path is followed. See also the accompanying animation (68396_06.avi [11.0MB]).

where 14 correct switches are performed for parameters (5.1) and perturbations $\epsilon=0.2$, $\eta=10^{-4}, \delta=0$ with $T=85.0$. (Again the same notation is used as in Figure 8 and the initial condition is at the three-cluster state $s_{1}=S_{\text {yywbb. }}$ ) Comparing Figure 17 to Figure 11 the same sequence of impulsive inputs are provided (red stars in panel (a)) and the system follows the same randomly chosen path (panel (b)). Even though relatively high noise is applied in Figure 17, the switching pattern is reliably followed.

We test the reliability of switching by following random paths of the network when impulses of amplitude $\epsilon$ are provided with period $T$ as in section 4.1. The error rate $E$ is obtained (from 2000 switches again) when varying the input period $T$ for several different values of noise strength $\eta$ and fixed input amplitude $\epsilon$. When the input amplitude $\epsilon$ is above some threshold, i.e., $\epsilon>\epsilon_{\mathrm{c}}\left(\epsilon_{\mathrm{c}} \approx 0.18\right.$ for parameters (5.1)), then there exist ranges of input period where reliable switching is possible as shown in Figure 18 by regions where $E \approx 0$. If the input amplitude is below the threshold, i.e., $\epsilon<\epsilon_{\mathrm{c}}$, then no switches can be triggered by the input so the error rate $E(T, \epsilon, \eta, \delta) \equiv 1$.

Figure 18 shows that for noise strength $\eta \ll \epsilon$ the error rate $E \approx 0$ is achievable when $T$ exceeds some critical period. That is, reliable switching is possible when a sufficiently long time is provided to travel along the connections described above. However, there also exists a window of input period in Figure 18 which corresponds to the case when the system gets close to a symmetrical copy of the subspace (3.11) but it is not in close vicinity of the three-cluster state embedded in the subspace. There exist regions in the subspace (e.g., the region around $(-0.95,0.95,-0.9)$ in Figure $15(\mathrm{~b}))$ where the provided inputs effectively place the system "on the other side" of the stable manifold of the symmetric copy of the cluster state $S_{\text {yywlb }}$ or $S_{\text {yywbl }}$ and thus lead to correct switches. 


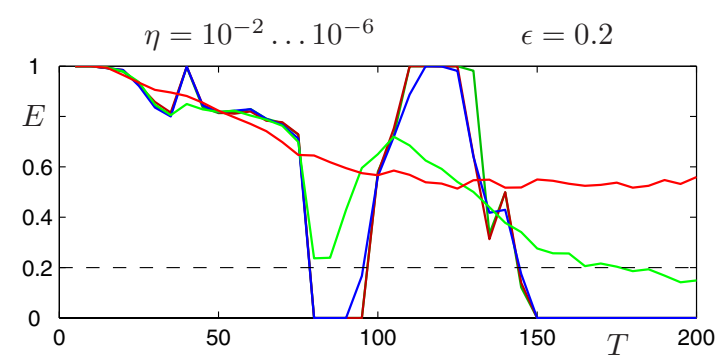

Figure 18. The error rate $E$ is shown as a function of the input period $T$ for the excitable network with parameters (5.1) when several different values of the noise strength $\eta$ are considered for fixed input amplitude $\epsilon$ and no detuning $\delta=0$. Observe that the boundaries where the error rate rapidly drops do not move as the noise strength is varied (except for strong noise).

The boundaries of these low error rate regions do not move much when the noise strength is varied except for very strong noise which increases the error rate for any $T$. This behavior can be explained by the stability of the three-cluster states and by the fact that $\epsilon$ is close to $\epsilon_{\mathrm{c}}$, that is, $\epsilon \gtrsim \epsilon_{\mathrm{c}}$. Small noise $\eta \ll \epsilon_{\mathrm{c}}$ does not provide enough perturbation to excite a switch between three-cluster states, while large enough noise $\eta>\epsilon_{\mathrm{c}}$ violates the condition $\eta \ll \epsilon$; that is, reliable switching is not expected for any $T$.

6. Conclusions. We investigated a globally coupled phase oscillator system as a simple model for a highly connected neural system. We generalized the coupling considered in $[2,13$, 17] and found an attracting robust heteroclinic network between partially synchronized threecluster states of saddle type. A graph representation of the network was given that can be used to explain much of its dynamical behavior, and we studied its response to perturbations such as noise, frequency detuning, and impulsive inputs, each of which can drive the system around the heteroclinic network. One may interpret this dynamics as a form of winnerless competition $[1,6,23]$ between three-cluster states since each of these states may be visited when tracking around the heteroclinic network but no state is an attractor for the system.

We found that the characteristic switching time between the three-cluster states is inversely proportional to the logarithm of the magnitude of perturbations. Broadly speaking, the bigger the perturbation, the faster the system "reacts" to it. We demonstrated that it is possible to reliably navigate the system along any chosen path on the heteroclinic network by providing small impulsive inputs to the appropriate oscillators, i.e., to perform finite-state computation as in [3]. Moreover, this can be done accurately in the presence of background noise and frequency detuning if the input amplitude dominates the noise strength and the detuning magnitude for a suitable range of input periods determined by these quantities. The boundaries of this range may be computed by considering the eigenvalues of the three-cluster states. In contrast, a steady input to the system (i.e., frequency detuning) resulted in a reduced network with attracting cyclic paths corresponding to a spatiotemporal output of the system.

We illustrated that on changing parameters in the coupling one can stabilize the threecluster states at a pitchfork bifurcation. This results in a robust network of excitable states with the same graph structure as the heteroclinic network. Hence, it is still possible to navigate around this excitable network by applying finite size impulsive inputs to the appropriate oscillators. 


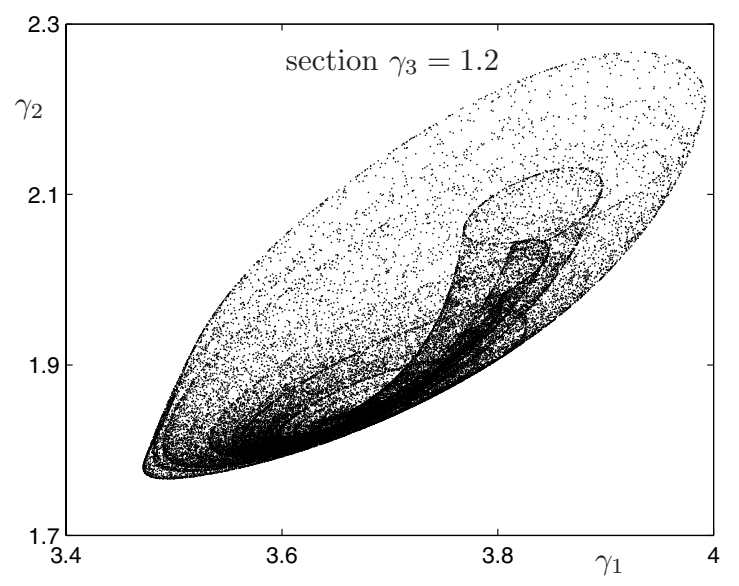

Figure 19. Chaotic attractor through a Poincaré section taken at $\gamma_{3}=\theta_{3}-\theta_{5}=1.2$ by projecting into the plane of $\gamma_{1}=\theta_{1}-\theta_{5}$ and $\gamma_{2}=\theta_{2}-\theta_{5}$. The parameters (7.1) are considered and no external perturbation is added $\epsilon=\eta=\delta=0$.

The studied dynamics shows that complex phase clustering can occur by spontaneous symmetry breaking in globally coupled systems, even in the absence of any inhomogeneity. Both the heteroclinic network (which is sensitive to arbitrarily small inputs) and the excitable network (which is only sensitive to finite amplitude inputs) can be used to represent the dynamics of systems that are sensitive to inputs but robust against background noise and imperfections. In these systems encoding via phase differences can be used for reliable finitestate computation as well as for producing spatiotemporal codes from steady inputs [23]. These features mimic the information processing capabilities of relating neural systems, such as the antennal lobes of insects [11]. In these neural systems the encoding is not in average frequencies of the oscillators but in their phases; i.e., effectively it is a form of spike-time coding.

7. Discussion of future directions. In this paper a region of parameter space was studied for the model (2.1), (2.2) of $N=5$ oscillators. The significance of $N=5$ is that this is the smallest number of oscillators that permits nontrivial cluster formations and switching between them [4]. Although the dynamics discussed for $N=5$ is robust, it does raise further questions:

(i) What other robust dynamics are possible for this system?

(ii) How does the dynamics change when the number of oscillators $N$ is increased?

Concerning (i), we illustrate that in the vicinity of the explored parameter regime there is chaotic behavior. Recall that to the right of the resonance bifurcation in Figure 14 the heteroclinic network is unstable. This unstable network may coexist with a chaotic attractor at the same parameter values. Figure 19 depicts a chaotic attractor for

$$
r=0.2, \quad \alpha=1.6, \quad \beta=-1.58, \quad \omega=1.0
$$

near to (3.1) in the unperturbed case $\epsilon=\eta=\delta=0$. The chaotic attractor is shown in a Poincaré section projected into the $\left(\gamma_{1}, \gamma_{2}\right)$-plane when $\gamma_{3}=1.2$; many symmetrical copies of 
the same attractor coexist in phase space. We believe this is the first example of a chaotic attractor in a system of globally coupled identical phase oscillators with no detuning $(\delta=0)$. (For example, the chaos observed in [20] for $N \geq 4$ oscillators of the Kuramoto system requires large detuning in relation to the coupling strength.)

Concerning (ii), preliminary investigations show that systems of $N>5$ phase oscillators also possess very rich dynamics including heteroclinic and excitable networks and chaotic attractors. In particular, for parameters (3.1) and moderate odd numbers of oscillators $N=7,9$ the only apparent attractor is a robust heteroclinic network of three-cluster states of type $((N-1) / 2,1,(N-1) / 2)$. These are connected analogously to section 3 for $N=5$ : one of the clusters of $(N-1) / 2$ oscillators is unstable to perturbations while the other is stable. These cluster states are well separated in phase space (the detection tolerance $\xi$ may be kept the same when $N$ is increased). We expect that most of the considerations in this paper will therefore follow through for these cases, except that the number of available three-cluster states/nodes and heteroclinic connections/directed edges are much larger (e.g., 140 states and 420 connections are available for $N=7$, while 630 states and 2520 connections are available for $N=9$ ). This combinatorial growth makes a thorough analysis much more difficult in the cases of $N>5$. Note that for other parameters we have found heteroclinic networks between cluster states of other types. However, in order to find a certain $\ell$-cluster state, more Fourier harmonics may need to be included in the coupling function. These preliminary results predict that the number of available cluster states can be enormously large for real neural ensembles.

Appendix A. Constants for eigenvalues and eigenvectors. The real part of (3.7) can be expressed as

$$
\mu=\frac{1}{10}\left(g^{\prime}(\chi)+2 g^{\prime}(-\chi)+g^{\prime}(\psi)+2 g^{\prime}(-\psi)+2 g^{\prime}(\chi-\psi)+2 g^{\prime}(\psi-\chi)\right),
$$

while its imaginary part contains

$$
\begin{aligned}
\nu=-\mu^{2}+\frac{4}{100}( & g^{\prime}(\chi) g^{\prime}(\psi)+2 g^{\prime}(\chi) g^{\prime}(-\psi)+2 g^{\prime}(-\chi) g^{\prime}(\psi) \\
& +2 g^{\prime}(\chi-\psi)\left(g^{\prime}(\psi)+2 g^{\prime}(-\chi)+2 g^{\prime}(-\psi)\right) \\
& \left.+2 g^{\prime}(\psi-\chi)\left(g^{\prime}(\chi)+2 g^{\prime}(-\chi)+2 g^{\prime}(-\psi)\right)\right) .
\end{aligned}
$$

The real parts of the components of (3.9) are

$$
\begin{aligned}
r_{\mathrm{y}}=-\frac{1}{4 Q}( & N_{1}\left(g^{\prime}(\chi)-g^{\prime}(\psi)-2 g^{\prime}(-\chi)-2 g^{\prime}(-\psi)+2 g^{\prime}(\chi-\psi)+2 g^{\prime}(\psi-\chi)\right) \\
& \left.-4 g^{\prime}(\chi) g^{\prime}(-\psi) g^{\prime}(\psi-\chi)+4 g^{\prime}(-\chi) g^{\prime}(\psi) g^{\prime}(\chi-\psi)\right) \\
r_{\mathrm{b}}=\frac{1}{4 Q}( & N_{3}\left(-g^{\prime}(\chi)+g^{\prime}(\psi)-2 g^{\prime}(-\chi)-2 g^{\prime}(-\psi)+2 g^{\prime}(\chi-\psi)+2 g^{\prime}(\psi-\chi)\right) \\
& \left.+4 g^{\prime}(\chi) g^{\prime}(-\psi) g^{\prime}(\psi-\chi)-4 g^{\prime}(-\chi) g^{\prime}(\psi) g^{\prime}(\chi-\psi)\right)
\end{aligned}
$$

Copyright $@$ by SIAM. Unauthorized reproduction of this article is prohibited. 
while the imaginary parts are

$$
\begin{aligned}
& p_{\mathrm{y}}=-\frac{1}{4 Q} N_{1} \sqrt{P}, \\
& p_{\mathrm{b}}=\frac{1}{4 Q} N_{3} \sqrt{P},
\end{aligned}
$$

where

$$
\begin{aligned}
& N_{1}=g^{\prime}(\chi) g^{\prime}(-\psi)+2 g^{\prime}(\chi-\psi)\left(g^{\prime}(-\chi)+g^{\prime}(-\psi)\right), \\
& N_{3}=g^{\prime}(-\chi) g^{\prime}(\psi)+2 g^{\prime}(\psi-\chi)\left(g^{\prime}(-\chi)+g^{\prime}(-\psi)\right),
\end{aligned}
$$

and

$$
\begin{aligned}
& P=-\left(g^{\prime}(\chi)-g^{\prime}(\psi)\right)^{2}-4\left(g^{\prime}(-\chi)+g^{\prime}(-\psi)\right)^{2}-4\left(g^{\prime}(\chi-\psi)+g^{\prime}(\psi-\chi)\right)^{2} \\
&-4\left(g^{\prime}(\chi)-g^{\prime}(\psi)\right)\left(g^{\prime}(-\chi)-g^{\prime}(-\psi)\right)-4\left(g^{\prime}(\chi)-g^{\prime}(\psi)\right)\left(g^{\prime}(\chi-\psi)-g^{\prime}(\psi-\chi)\right) \\
&+8\left(g^{\prime}(-\chi)+g^{\prime}(-\psi)\right)\left(g^{\prime}(\chi-\psi)+g^{\prime}(\psi-\chi)\right), \\
& Q= g^{\prime}(-\chi) g^{\prime}(-\psi)\left(g^{\prime}(\chi)-g^{\prime}(\psi)\right)+\left(g^{\prime}(-\chi)+g^{\prime}(-\psi)\right)\left(2 g^{\prime}(-\chi) g^{\prime}(\chi-\psi)\right. \\
&\left.-2 g^{\prime}(-\psi) g^{\prime}(\psi-\chi)\right) .
\end{aligned}
$$

Copyright (c) by SIAM. Unauthorized reproduction of this article is prohibited. 


\section{Appendix B. The operator $O_{p}$.}

Table 2

The effects of operator $O_{p}, p=1, \ldots, 5$, in (3.13) on the three-cluster states $s_{i}, i=1, \ldots, 30$. The resulting states are shown only when $i \neq j$ for $O_{p}\left(s_{i}\right)=s_{j}$; in all other cases $O_{p}\left(s_{i}\right)=\left(s_{i}\right)$.

\begin{tabular}{|c|c|c|c|c|c|}
\hline & $O_{1}\left(s_{i}\right)$ & $\mathrm{O}_{2}\left(s_{i}\right)$ & $\mathrm{O}_{3}\left(s_{i}\right)$ & $\mathrm{O}_{4}\left(s_{i}\right)$ & $O_{5}\left(s_{i}\right)$ \\
\hline$s_{1}$ & & & & $s_{12}$ & $s_{13}$ \\
\hline$s_{2}$ & $s_{20}$ & $s_{30}$ & & & \\
\hline$s_{3}$ & & $s_{27}$ & & & $s_{17}$ \\
\hline$s_{4}$ & $s_{23}$ & & & $s_{10}$ & \\
\hline$s_{5}$ & & $s_{26}$ & & $s_{7}$ & \\
\hline$s_{6}$ & $s_{22}$ & & & & $s_{15}$ \\
\hline$s_{7}$ & $s_{19}$ & & & & $s_{18}$ \\
\hline$s_{8}$ & & $s_{26}$ & $s_{6}$ & & \\
\hline$s_{9}$ & $s_{23}$ & & $s_{3}$ & & \\
\hline$s_{10}$ & & $s_{29}$ & & & $s_{16}$ \\
\hline$s_{11}$ & & & $s_{2}$ & & $s_{13}$ \\
\hline$s_{12}$ & $s_{21}$ & $s_{28}$ & & & \\
\hline$s_{13}$ & $s_{24}$ & $s_{25}$ & & & \\
\hline$s_{14}$ & & & $s_{2}$ & $s_{12}$ & \\
\hline$s_{15}$ & & $s_{29}$ & & $s_{9}$ & \\
\hline$s_{16}$ & $s_{22}$ & & $s_{5}$ & & \\
\hline$s_{17}$ & $s_{19}$ & & & $s_{8}$ & \\
\hline$s_{18}$ & & $s_{27}$ & $s_{4}$ & & \\
\hline$s_{19}$ & & $s_{30}$ & $s_{1}$ & & \\
\hline$s_{20}$ & & & & $s_{8}$ & $s_{18}$ \\
\hline$s_{21}$ & & & $s_{5}$ & & $s_{15}$ \\
\hline$s_{22}$ & & $s_{28}$ & & $s_{11}$ & \\
\hline$s_{23}$ & & $s_{25}$ & & & $s_{14}$ \\
\hline$s_{24}$ & & & $s_{3}$ & $s_{10}$ & \\
\hline$s_{25}$ & & & $s_{6}$ & $s_{7}$ & \\
\hline$s_{26}$ & $s_{24}$ & & & & $s_{14}$ \\
\hline$s_{27}$ & $s_{21}$ & & & $s_{11}$ & \\
\hline$s_{28}$ & & & $s_{4}$ & & $s_{17}$ \\
\hline$s_{29}$ & $s_{20}$ & & $s_{1}$ & & \\
\hline$s_{30}$ & & & & $s_{9}$ & $s_{16}$ \\
\hline
\end{tabular}

Acknowledgments. The authors thank Roman Borisyuk for discussions on neurodynamics and Stephen Coombes and Sebastian Wieczorek for asking perceptive questions.

\section{REFERENCES}

[1] V. S. Afraimovich, M. I. Rabinovich, And P. Varona, Heteroclinic contours in neural ensembles and the winnerless competition principle, Internat. J. Bifur. Chaos Appl. Sci. Engrg., 14 (2004), pp. 1195-1208.

[2] P. Ashwin And J. Borresen, Encoding via conjugate symmetries of slow oscillations for globally coupled oscillators, Phys. Rev. E (3), 70 (2004), 026203.

[3] P. Ashwin And J. Borresen, Discrete computation using a perturbed heteroclinic network, Phys. Lett. A, 347 (2005), pp. 208-214.

Copyright $@$ by SIAM. Unauthorized reproduction of this article is prohibited. 
[4] P. Ashwin, O. Burylko, Yu. Maistrenko, and O. Popovych, Extreme sensitivity to detuning for globally coupled phase oscillators, Phys. Rev. Lett., 96 (2006), 054102.

[5] P. Ashwin And J. W. Swift, The dynamics of $n$ weakly coupled identical oscillators, J. Nonlinear Sci., 2 (1992), pp. 69-108.

[6] P. Ashwin And M. Timme, When instability makes sense, Nature, 436 (2005), pp. 36-37.

[7] E. Brown, P. Holmes, And J. Moenlis, Globally coupled oscillator networks, in Perspectives and Problems in Nonlinear Science: A Celebratory Volume in Honor of Larry Sirovich, K. Sreenivasan, E. Kaplan, and J. Marsden, eds., Springer, New York, 2003, pp. 183-215.

[8] E. Brown, J. Moehlis, And P. Holmes, On the phase reduction and response dynamics of neural oscillator populations, Neural Computation, 16 (2004), pp. 673-715.

[9] S.-N. Chow, B. Deng, And B. Fiedler, Homoclinic bifurcation at resonant eigenvalues, J. Dynam. Differential Equations, 2 (1990), pp. 177-244.

[10] E. J. Doedel, A. R. Champneys, T. F. Fairgrieve, Yu. A. Kuznetsov, B. Sandstede, and X. WANG, AUTO97: Continuation and Bifurcation Software for Ordinary Differential Equations, Tech. report, Department of Computer Science, Concordia University, Montreal, Canada, 1997, http:// indy.cs.concordia.ca/auto/.

[11] C. G. Galizia, S. Sachse, A. Rappert, and R. Menzel, The glomerular code for odor representation is species specific in the honeybee apis mellifera, Nature Neuroscience, 2 (1999), pp. 473-478.

[12] M. Golubitsky and I. N. Stewart, The Symmetry Perspective: From Equilibrium to Chaos in Phase Space and Physical Space, Progr. Math. 200, Birkhäuser Boston, Boston, 2003.

[13] D. Hansel, G. Mato, And C. Meunier, Clustering and slow switching in globally coupled phase oscillators, Phys. Rev. E, 48 (1993), pp. 3470-3477.

[14] D. Hansel, G. Mato, And C. Meunier, Phase dynamics for weakly coupled Hodkin-Huxley neurons, Europhys. Lett., 23 (1993), pp. 367-372.

[15] B. Hasselblatt And A. KatoK, The development of dynamics in the 20th century and the contribution of Jürgen Moser, Ergodic Theory Dynam. Systems, 22 (2002), pp. 1343-1364.

[16] E. M. Izhikevich, Dynamical Systems in Neuroscience: The Geometry of Excitability and Bursting, MIT Press, Cambridge, MA, 2007.

[17] H. Kori And Y. Kuramoto, Slow switching in globally coupled oscillators: Robustness and occurrence through delayed coupling, Phys. Rev. E, 63 (2001), 046214.

[18] M. KRuPA, Robust heteroclinic cycles, J. Nonlinear Sci., 7 (1997), pp. 129-176.

[19] Y. Kuramoto, Self-entrainment of a population of coupled non-linear oscillators, in International Symposium on Mathematical Problems in Theoretical Physics, Lecture Notes in Phys. 39, H. Araki, ed., Springer-Verlag, New York, 1975, pp. 420-422.

[20] Yu. Maistrenko, O. Popovych, And M. Hasler, On strong and weak chaotic partial synchronization, Internat. J. Bifur. Chaos Appl. Sci. Engrg., 10 (2000), pp. 179-203.

[21] K. OKUDA, Variety and generality of clustering in globally coupled oscillators, Phys. D, 63 (1993), pp. $424-436$

[22] G. Orosz And G. StÉPÁn, Subcritical Hopf bifurcations in a car-following model with reaction-time delay, Proc. R. Soc. Lond. Ser. A Math. Phys. Eng. Sci., 462 (2006), pp. 2643-2670.

[23] M. I. Rabinovich, P. Varona, A. I. Selverston, and H. D. I. Abarbanel, Dynamical principles in neuroscience, Rev. Modern Phys., 78 (2006), pp. 1213-1265.

[24] E. Stone And P. Holmes, Random perturbations of heteroclinic attractors, SIAM J. Appl. Math., 50 (1990), pp. 726-743.

Copyright (C) by SIAM. Unauthorized reproduction of this article is prohibited. 
Copyright of SIAM Journal on Applied Dynamical Systems is the property of Society for Industrial \& Applied Mathematics and its content may not be copied or emailed to multiple sites or posted to a listserv without the copyright holder's express written permission. However, users may print, download, or email articles for individual use. 